\title{
Targeting SARS-CoV-2 infection through CAR T cells and bispecific T cell engagers
}

Mikail Dogan¹, Lina Kozhaya ${ }^{1}$, Lindsey Placek${ }^{1}$, Fatih Karabacak ${ }^{1}$, Mesut Yigit $^{1,2}$ and Derya Unutmaz ${ }^{1,3, \#}$

1 Jackson Laboratory for Genomic Medicine, Farmington, Connecticut, USA.

${ }^{2}$ Current address: Acibadem Mehmet Ali Aydinlar University School of Medicine, Istanbul, Turkey

${ }^{3}$ Department of Immunology, University of Connecticut School of Medicine, Farmington, CT USA.

\# Corresponding author E-mail: derya@mac.com 


\section{Summary}

Despite advances in antibody treatments and vaccines, COVID-19 caused by SARS-CoV-2 infection remains a major health problem resulting in excessive morbidity and mortality and the emergence of new variants has reduced the effectiveness of current vaccines. Here, we engineered SARS-CoV-2 Spike protein-specific CAR, using extracellular region of ACE2, and expressed in primary CD8 T cells. Alternatively developed bispecific T cell engager molecules combining ACE2 with anti-CD3 (ACE2-Bite) to target infected cells and the virus. Both, ACE2 CAR T cells and ACE2-Bite selectively killed Spike protein-expressing targets. In addition, ACE2Bites neutralized pseudoviruses and activated T cells with Spike proteins, both wild type and in those with mutations derived from variants such as Alpha, Beta and Delta. These approaches have the potential to render them fool-proof for current or future spike mutations. Taken together, the approaches we report here could be considered as future therapeutic strategies during early and late COVID-19 patients.

\section{Keywords: SARS-CoV-2, COVID-19, ACE2 CAR, ACE2-Bite}




\section{Introduction}

Worldwide, Coronavirus disease 2019 (COVID-19) caused by SARS-CoV-2 infection has caused close to 5 million deaths and a chronic debilitating condition called Post-Acute COVID-19 Syndrome (PACS) in many millions more. An unprecedented effort by researchers around the world has resulted in the development of a spectrum of preventative and therapeutic approaches at an extraordinary speed. Vaccines focused on virus Spike protein (such as messenger RNA vaccines, non-replicating vector vaccines, and virus-like particle vaccines) are highly efficient in preventing infection (Wu et al., 2021). Several therapeutic developments, such as synthetic neutralizing antibodies, monoclonal antibodies to Spike protein (Baum et al., 2020) (Gottlieb et al., 2021), (Guimaraes et al., 2021; Marconi et al., 2021) and immunomodulators such as corticosteroids (Gandhi et al., 2020; Group et al., 2021), anti-IL-6 (Cellina et al., 2020) , anti-IL-1 (Huet et al., 2020) and Interferon- $\beta-1$ a agents (Monk et al., 2021) were shown to have a range of treatment efficacy from non-effective to highly promising. Some of those treatments were repurposed to focus on blocking viral entry while others were used to control the hyperinflammatory immune response during the disease. Beyond antibody therapies, specific SARS-CoV-2 immunomodulators have not been developed yet are needed as specific antibodies potentially lose their effectiveness due to new variants. Therefore, the development of treatment approaches that remain effective against SARS-CoV-2 variants is of great interest.

SARS-CoV-2 uses its Spike protein to bind to the key host receptor Angiotensin-Converting Enzyme 2 (ACE2) on target cell surface for cell entry (Hoffmann et al., 2020) and mutations in Spike protein result in higher affinity of the virus to ACE2 (Barton et al., 2021) and/or a better escaping mechanism from the immune system (Harvey et al., 2021). Following the cell entry, SARS-CoV-2 generates viral components by taking over the protein synthesis machinery of the 
host cell and displays Spike protein on the cell membrane (Ou et al., 2020). Using ACE2 molecule to target these Spike-expressing infected cells could be an effective strategy in preventative and therapeutic approaches to COVID-19 in the future since ACE2 receptor would have to be compatible to the binding of forthcoming mutant Spike proteins.

Here, we used a synthetic biology approach to engineer primary human CD8 T cells to express Spike protein-specific chimeric antigen receptors (ACE2 CAR or anti-Spike CAR) with ACE2 or anti-Spike antibody on the extracellular domain to target SARS-CoV-2 infected cells. We also engineered a novel ACE2/anti-CD3 bispecific T cell engager antibody (ACE2-Bite) to target both SARS-CoV-2 infected cells and the SARS-CoV-2 virus. Bispecific T cell engager antibodies (Bites) are engineered chimeric molecules that are designed to bind to CD3 on T cells via an antiCD3 single chain variable fragment (ScFv) and to a target cell via a target-specific molecule. Upon bridging the $\mathrm{T}$ cells with a target cell, Bites trigger $\mathrm{T}$ cell activation and subsequent target cell cytotoxicity. CAR-T and bispecific T cell engager antibody approaches we developed in our study are similar to methods targeting cancer cells, several of which have been approved for treatment (Ahmad et al., 2020).

We show that ACE2-CAR and anti-Spike CAR-expressing CD8 T cells became activated and selectively kill different types of target cells expressing SARS-CoV-2 Spike protein on their surface. The ACE2-Bite antibodies also led to T cell activation in the presence of Spike expressing targets, and mediated cytotoxicity to these targets. In addition, ACE2-Bite antibodies acted as a decoy receptor for wild type and mutated Spike pseudotyped virus and neutralized them regardless of their mutations. Taken together, these results suggest that the novel chimeric antigen receptors and bispecific antibodies may be used to redirect cytotoxic immune cells towards SARS-CoV-2 infected host cells and to neutralize variant strains of the virus. 


\section{Results}

\section{Development of SARS-CoV-2 specific synthetic CARs expressed in T cells.}

We sought to develop a system to test whether we can target cells that express cell surface SARS-CoV-2 Spike protein on their cell surface during the infection (Cattin-Ortola et al., 2021) (Figure 1A) employing effector human T cells engineered to express CAR molecules that can recognize the Spike protein on cell surface. For that, we transfected the cells with a plasmid containing a full-length wild-type Spike protein gene under CMV promoter (Figure 1B). 72 hours later cells were stained with a recombinant ACE2-Fc protein followed by an anti-Fc antibody to detect surface Spike protein expression and compared to control Vesicular stomatitis Virus G (VSVG) plasmid transfected cells. 293 cells transfected with full-length Spike protein plasmid displayed cell surface Spike expression indicating Spike protein indeed can be localized to the cell membrane despite its ERRS domain (Figure 1C). We then established a target 293 cell line that stably expressed both the Spike protein and Green Fluorescent Protein (GFP) as a reporter. Further, to enhance cell surface spike protein expression as shown in previous studies (Dieterle et al., 2020; Duan et al., 2020), we deleted the ERRS domain. 72 hours after the transduction, engineered 293 cells were stained for their Spike expression, and flow cytometry analysis showed a co-expression of Spike and GFP in a high percentage of cells (Figure 1D).

Next, we designed lentivector constructs containing ACE2 CAR or anti-Spike CAR cassettes followed by an Internal Ribosomal Entry Site (IRES) and Red Fluorescent Protein (RFP) and transduced human primary CD8 T cells (Figure 1E) as previously described (Wan et al., 2013). 
ACE2 CAR and anti-Spike CAR constructs comprised of CD8 alpha signal peptide, ACE2 extracellular domain (ECD) or anti-Spike ScFv, respectively, and intracellular 41BB co-stimulatory domain (CSD), and CD3ろ (zeta) signaling domains (Figure 1E). An Anti-CD19 CAR-RFP lentiviral construct was also designed to be used as a control. CD8 T cells were then activated and transduced with these lentiviruses encoding the CAR constructs and expanded in IL-2 for 10-12 days. CD8 T cells engineered with ACE2 CAR and anti-Spike CAR constructs expressed these on cell surface, which also correlated with RFP reporter expression (Figure 1F).

\section{Cytotoxicity assays with ACE2 CAR and anti-Spike CAR expressing T cells.}

We then co-cultured Spike+ target cell line and effector T cells expressing CARs and measured the cytotoxicity activity of the T cells (Figure 2A). Briefly, after the $\sim 2$-week proliferation of CART cells, the cells were co-cultured for 72 hours with Spike-expressing target cells at different effector to target ratios. The CD8+ T cells were then stained with anti-CD25 to determine their activation. Target cells were identified via GFP, which was co-expressed with Spike protein. Both ACE2 CAR and anti-Spike CAR-T cells became highly activated and killed the Spike+ 293 cells whereas control anti-CD19 CAR-T cells were neither activated nor showed any cytotoxicity (Figure 2B, 2C).

We next tested whether ACE2 CAR and anti-Spike CAR-T cells can kill Spike-expressing human B cell line, which can also be used as positive control using anti-CD19 CAR T cells. ACE2 CAR and anti-Spike CAR-T cells killed Spike-expressing B cells as efficiently as 293 cells, indicating that different cell types infected with SARS-CoV-2 can be targeted using these novel CAR-T cells (Figure 2D). In addition, ACE2 CAR and anti-Spike CAR-T cells did not show cytotoxicity to GFPexpressing, Spike-negative control targets and were also not activated, showing a selective Spike protein-mediated activation and killing (Supplementary Figure 1). 


\section{Development of bispecific antibodies to mobilize and activate $T$ cells against}

\section{SARS-CoV-2 Spike protein expressing target cells.}

Currently, the CAR-T cell immunotherapy procedure requires a meticulous process of collecting cells from patients, engineering them in a GMP environment, re-infusion, and extensive clinical follow-up of the patients (Sterner and Sterner, 2021). As such this may not be very practical for treatment of COVID-19 patients. As an alternative, we also engineered bispecific antibodies (Bites) as T cell activators, consisting of an anti-CD3 scFv fused with the extracellular domain of ACE2 to redirect CD3 T cells to SARS-CoV-2 infected cells (Figure 3A). The ACE2-Bite cassette consisted of ACE2 signal peptide, ACE2 extracellular domain, a linker peptide, an anti-CD3 antibody single-chain variable fragment, a His-Tag, and a Hemagglutinin (HA) Tag (Figure 3B). ACE2-Bite was produced in suspension 293 cells as described in methods. The supernatant from these cells were then filtered to eliminate molecules smaller than $30 \mathrm{kDa}$, which also resulted in 30-fold concentration of ACE2-Bite proteins. The supernatant of wild-type suspension 293 cells was also collected and filtered/concentrated to be used as a control.

To test the correct folding of the recombinant ACE2-Bite protein, we developed a fluorescent bead-based ACE2-Bite detection assay in which the fluorescent beads were coated with Receptor Binding Domain of SARS-CoV-2 Spike protein (S-RBD) and ACE2-Bite molecules captured by SRBD beads were detected via a recombinant CD3-Fc molecule which was then stained with an anti-Fc antibody (Figure $3 C$ ). A recombinant ACE2-Fc molecule was used as a positive control since ACE2 part could bind to S-RBD on the surface of beads and anti-Fc antibody could recognize the Fc part of ACE2-Fc. ACE2-Bite detection assay showed that detected ACE2-Bite levels (1:10) were comparable to control ACE2-Fc concentration (3 $\mu \mathrm{g} / \mathrm{mL})$ (Figure 3D) and correlated with ACE2-Bite secreting 293 cell density (Supplementary Figure 2). We also 
confirmed that ACE2-Bite concentration protocol functioned as intended and increased the ACE2Bite concentration by an order of magnitude (Supplementary Figure 2).

We then tested the ACE2-Bite binding on human primary CD8 T and Spike-expressing target cells. For this, ACE2-Bite and wild-type supernatants were added to a B cell line (T2 cells) which was engineered to express Spike/GFP and primary human CD8 T cells. The cells combined with ACE2-Bite or control supernatants were then stained for HA Tag on their surface. Spike/GFP coexpressing T2 cells and CD3 expressing primary human CD8 T cells combined with ACE2-Bites were stained positive for HA Tag, suggesting Spike specific binding to ACE2 fragment and CD3 specific binding to Anti-CD3 fragment. (Figure 3E).

We then performed a cytotoxicity assay to test the ability of ACE2-Bites to trigger primary human T cell activation. Human CD8 T cells were co-cultured with Spike-expressing or control 293 cells in the presence of ACE2-Bite or control supernatants. 2 days later cells were collected and stained for their CD8 and CD25 expression. GFP expressed by control and Spike lentivectors was used to identify the target cells. Indeed, resting human T cells became activated and were cytotoxic only in the presence of ACE2-Bite supernatant and Spike-expressing targets, suggesting Spikespecific T-cell activation functionality of ACE2-Bites (Figure 3F).

\section{Determining function of ACE2-Bite on mutated Spike proteins.}

A major advantage of targeting the SARS-CoV-2 Spike protein through its receptor ACE2 (ACE2Bite) is that this approach is less affected by antibody escape mutations, as mutated Spike proteins would still need to interact with ACE2. In fact, it is conceivable that variants with increased affinity to ACE2 would bind better to ACE2-Bite, possibly improving its efficacy. 
To test this with an ACE2-Bite/Spike binding assay, we transfected 293 cells with plasmids to express 7 different mutant Spike proteins (Table 1) and determined the ACE2-Bite binding to these Spike proteins (Figure 4A). 3 days after the transfection, the cells were collected and costained with ACE2-Bite and an anti-Spike antibody and analyzed via flow cytometry. The ACE2Bite/Spike binding assay revealed that the mean fluorescent intensity of cells stained with ACE2Bite under anti-Spike antibody-stained cell population increased with some of the mutations of the Spike protein with the exception of K417N (Figure 4B). Other studies also reported the weakening effect of K417N mutation on the affinity of Spike to ACE2 (Barton et al., 2021) (Laffeber et al., 2021). Cell cultures with these transfected 293 cells in the presence of ACE2-Bite, highly efficiently activated CD8 T cells, regardless of the type of Spike protein mutations, implicating potential pan-SARS-CoV-2 effectivity of ACE2-Bite approach (Figure 4C).

\section{ACE2-Bite molecules neutralize Spike-expressing lentiviruses.}

In addition to bridging infected cells to activate T cells, we reasoned that ACE2-Bite may also neutralize SARS-CoV-2 by binding to Spike proteins on the virus. To test this, we generated a set of lentiviruses pseudotyped with 7 different Spike proteins and determined ACE2-Bite binding to Spike. Neutralization was determined by pre-culturing pseudotyped viruses with different dilutions of ACE2-Bite molecule and then adding to ACE2 expressing 293 cells as previously described (Dogan et al., 2021) (Figure 5A). We also incubated a recombinant ACE2-Fc molecule at different concentrations with Spike pseudotyped lentivirus as a positive control. The infection levels were determined 3 days post-infection based on the GFP expression of ACE2-expressing 293 cells. As shown in the representative experiment, ACE2-FC and ACE2-Bite molecules neutralized the Spike pseudotyped lentivirus (Figure 5B). Importantly, ACE2-Bite molecule was able to neutralize all of the mutant Spike encoding lentiviruses with similar efficiencies (Figure $\mathbf{5 C}$ ). These 
neutralization assays demonstrated that novel ACE2-Bite recombinant protein could also function as a decoy receptor against the virus.

\section{Discussion}

Despite advances in vaccine development, COVID-19 is still a major cause of morbidity and mortality in the USA and throughout the world. Rapid evolution of the virus is also a major concern, suggesting the need to develop novel effective treatment strategies, as SARS-CoV-2 specific targeted therapeutic approaches such as monoclonal antibody therapies can lose their effectiveness as new escape variants emerge. To mitigate or override these potential problems, here we utilized synthetic biology approaches, to develop synthetic molecules that can bridge $\mathrm{T}$ cells with SARS-CoV-2 infected cells, through recognition of cell surface expression of virus Spike protein and eliminate them through cytotoxic activity.

We first generated CD8 T cells expressing chimeric antigen receptors (CARs) specific to Spike protein with an anti-Spike antibody or ACE2 surface domain on the extracellular region and tested their effectiveness against different cell types expressing Spike proteins. In this assay, engineered CAR-T cells (anti-Spike CARs and ACE2 CARs) became activated and killed the Spikeexpressing target cells selectively. Although cancer cells have predominantly been the focus of adaptive cellular immunotherapies, studies have suggested that autoimmune and infectious diseases could also be targeted via such approaches (Maldini et al., 2018; Wraith, 2017). Several studies have employed or investigated CAR-T approach against infectious diseases, such as Human immunodeficiency virus (HIV) (Leibman et al., 2017; Mitsuyasu et al., 2000; Savoldo et al., 2011), Hepatitis B virus (HBV) (Kruse et al., 2018), Hepatitis C virus (HCV) (Sautto et al., 2016), Cytomegalovirus (CMV) (Full et al., 2010), Epstein-Barr virus (EBV) (Tang et al., 2014) and Aspergillus fumigatus (Kumaresan et al., 2014). While it is not practical to apply this approach 
during COVID-19, it is at least conceptually possible to develop CAR-T cells engineered to target infected cells as prophylaxis, in immune-compromised or elderly individuals who are in the highrisk category for mortality, as the lifespan of these cells could be many months to years. It may also be possible to generate off-the-shelf Spike-specific CAR-T cells by removing genes in T cells that can cause allogeneic reaction or graft versus host disease (Depil et al., 2020). This may also be an important proof-of-principle for other viral infections, current or future for which we do not yet have vaccines available (Bailey et al., 2019; Johnston and Fauci, 2008).

In this study, we also demonstrated that ACE2-Bite bispecific antibodies triggered effective CD8 T cell activation, which resulted in selective killing of Spike+ cells. Bispecific $T$ cell engagers (Bites) are already approved for use in humans, following the accelerated approval of Blinatumomab (CD19-CD3 Bite) in March 2018 for B-cell lymphomas (Kantarjian et al., 2017). Other studies also showed that Bites could be employed against Her2 (Yu et al., 2019), BCMA (Goldstein et al., 2020), EpCAM (Ferrari et al., 2015), EGFR (Lutterbuese et al., 2010), CD20 (Hosseini et al., 2020), and PDL1 (Horn et al., 2017) expressing cancers; and diseased cells infected by CMV (Brey et al., 2018) and HIV (Sung et al., 2015). Compared to current treatments (such as neutralizing antibodies or anti-virals) ACE2-Bite approach may potentially be effective both at early stages (as neutralizer of the virus entry) and later stages of the infection when antibody immune defenses are breached, and T cells become more important in restricting the spread of the virus in vivo. A major advantage of the ACE2-Bite approach is using ACE2, the key host receptor of SARS-CoV-2, as the Spike protein recognizing part of the bi-specific antibody. As shown in Figure 4, this allowed us to target many mutated Spike proteins from variants of concern, regardless of their mutations.

In addition to recognizing mutated Spike proteins, we found that ACE2 part of the ACE2-Bite functioned as a decoy receptor and neutralized the virus, preventing it from infecting the cells. 
The neutralization feature of the ACE2-Bite molecule is promising for its use as preventive treatment and its use would conceivably have synergistic effect with the cytotoxic effect by engaging T cells towards infected cells. Indeed, in line with our findings, a clinical study by Zoufaly et al. found that infusion of soluble recombinant human ACE2 molecule in a 45-year-old COVID19 patient resulted in dramatic decrease in viral copies in the patient plasma (Zoufaly et al., 2020). Other studies also demonstrated the neutralization capacity of soluble ACE2 molecule (Case et al., 2020; Glasgow et al., 2020; Monteil et al., 2020). Considering the immunity of ACE2 to the Spike mutations, both as a CD3 T cell redirecting molecule and a decoy receptor, the efficacy of the ACE2-Bite treatment is unlikely to be diminished by variants arising during COVID-19 pandemic or in possible future SARS pandemics.

If these approaches are developed as therapeutics, it will also be important to consider side effects of CAR-T cells or ACE2-Bite treatment, cytokine release syndrome and T cell exhaustion, similar to cancer focused CAR-T therapies (Subklewe, 2021). Although, we think this will be less likely given the tumor burden would be expected to be much greater compared to virus infected cells; overall, much fewer T cells would be stimulated during the infection. Another potential downside could be that the ACE2 in ACE2-Bite or ACE2 CAR may interact with its physiologic ligands and interfere with the renin-angiotensin system, although, although so far recombinant human ACE2 molecule has been tested in 89 patients with tolerable clinical outcomes (Haschke et al., 2013; Khan et al., 2017). Regardless, if this becomes a problem it can be prevented by mutating the carboxypeptidase activity of ACE2. Furthermore, downregulation of ACE2 levels due to SARS-CoV-2 binding during COVID-19 is associated with hyperactivation of renin-angiotensin system and injuries to the lung, kidneys, and heart (Silhol et al., 2020). Thus, ACE2-Bite or ACE2CAR may even have beneficial properties as a replacement to wild-type ACE2 activity during COVID-19. 
In conclusion, engineered CD8 T cells expressing Spike protein-specific chimeric antigen receptors and ACE2/anti-CD3 bispecific antibodies developed in this study could be used to target SARS-CoV-2 infected host cells and the virus itself, and may be alternative future therapeutic strategies for COVID-19.

\section{Acknowledgments}

The research in this study was supported by National Institute of Health (NIH) grant U19 Al142733-01 (DU) and Achelis and Bodman Foundation (DU). We thank Courtney Gunter and Sara Cassidy for critical reading and Mustafa Semih Elitok for advice on designing anti-Spike antibody ScFv proteins.

\section{Author Contributions}

M.D., L.K. and D.U. conceived and designed the experiments. M.D., L.K., L.P., F.K., and M.Y. carried out the experiments. M.D., L.K., and D.U. analyzed the data, drew illustrations, and prepared the figures. M.D. and D.U. wrote the manuscript.

\section{Declaration of Interests}

M.D. and D.U. are authors in a potential patent application. 


\section{Figure legends}

\section{Figure 1: Engineering human primary CD8 $\mathrm{T}$ cells to express CAR molecules targeting SARS-CoV-2 Spike protein expressing cells.}

(A) Illustration of Spike protein localization on the surface of SARS-CoV-2 infected cells and (B) of full-length SARS-CoV-2 Spike protein mRNA expressing plasmid including the Endoplasmic Reticulum Retention Signal (ERRS) of Spike protein on C terminal. (C) 293 cells transfected with full-length Spike protein (blue histogram) or with VSV-G as a negative control (red histogram) expressing vectors. The cells were stained with ACE2-Fc and anti-Fc-APC secondary antibody, flow cytometry data overlays are shown. (D) 293 cells transduced with a lentivirus encoding a truncated Spike protein gene without the ERRS domain and Green Fluorescent Protein (GFP) as a reporter. Transduced cells were stained with ACE2-Fc and anti-Fc-APC secondary antibody, representative flow cytometry data plots are shown. (E) Illustration of ACE2 CAR and anti-SARSCoV-2 Spike protein CAR constructs and their expression in CD8 T cells. A constitutive LTR promoter drives ACE2 or anti-Spike CAR and RFP genes separated by an Internal Ribosomal Entry Site (IRES). CAR constructs consist of CD8 alpha signal peptide, ACE2 or single chain variable fragment of an anti-Spike antibody, CD8 Hinge, CD8 transmembrane domain, 4-1BB (CD137) co-stimulatory domain and CD3 $\zeta$ domain. Lentiviruses containing CARs were used to transduce primary CD8 T cells. (F) Expression of CAR constructs on CD8 T cells. Activated and transduced CD8 T cells were expanded for 10-12 days and stained with SARS-CoV-2 S1 protein fused to mouse Fc, and anti-mouse Fc secondary antibody. Flow cytometry plots showing ACE2 or anti-Spike surface expression versus RFP are shown. Anti-CD19 CAR expressing CD8 T cells were used as control. The experiments were replicated several times with similar results. 
Figure 2: Cytotoxic activity of human primary CD8 T cells engineered to express ACE2 CAR or anti-Spike CAR

(A) Illustration of cytotoxicity assay against Spike-expressing target cells using ACE2 CAR or anti-Spike CAR expressing CD8 T cells as effector cells. (B) CAR engineered T cells cytotoxicity assays with Spike expressing 293 target cells at different Effector:Target ratios. CD8 T cells transduced with anti-CD19 CAR lentiviruses were used as control effector cells. Effector CD8 T cells were identified with CD8 staining while target cells were gated based on GFP (Spike) expression. Activation of effector cells and CAR expression were determined with CD25 expression after gating on CD8 T cells. (C) Percent cytotoxicity of ACE2 CAR (blue) and antiSpike CAR (purple) T cells normalized to anti-CD19 CAR-T cells at different Effector:Target ratios and using Spike-expressing 293 cells as the target. (D) CAR engineered T cells cytotoxicity assays with Spike-expressing target B cell line (T2 cells). Wild-type CD8 T cells were used as negative control and anti-CD19 CAR expressing CD8 T cells were used as positive control. Panels show representative experiments replicated with similar results.

\section{Figure 3: Functional ACE2/anti-CD3 bi-specific $\mathrm{T}$ cell engagers against}

\section{SARS-CoV-2}

(A). Illustration describing potential mechanism of action of ACE2-Bite. The extracellular domain (ECD) of ACE2 (blue) in ACE2-Bite binds to Spike protein (red) expressed on the surface of SARS-CoV-2 infected cells and the anti-CD3 fragment (orange) binds to CD3 molecule (purple) on T cells linking both cell types and inducing the activation of T cells which subsequently results in apoptosis of infected target cells. ACE2-Bite recombinant protein also contains a hemagglutinin (HA) tag at the $\mathrm{C}$ terminal. (B) Representation of ACE2-Bite construct and the protein production in 293 cells. A constitutive LTR promoter drives the expression of ACE2-Bite and RFP genes 
separated by an Internal Ribosomal Entry Site (IRES). ACE2-Bite cassette consists of ACE2 signal peptide (SP), ACE2 extracellular domain, a linker peptide, an anti-CD3 antibody singlechain variable fragment, a His-Tag, and a Hemagglutinin (HA) Tag. Lentiviruses expressing ACE2-Bite were used to transduce suspension 293 cells that produce and secrete ACE2-Bite protein in their culture supernatant. (C) Representation of the bead-based ACE2-Bite capture assay. Fluorescent beads coated with Spike-Receptor binding domain (S-RBD) were used to capture ACE2-Bite molecules which were detected via a recombinant CD3-Fc fusion protein and an anti-Fc antibody then subsequently analyzed via flow cytometry. ACE2-Fc molecules were also detected with S-RBD coated beads and anti-Fc antibody. (D) Detection of different concentrations of ACE2-Bite (1:10 and 1:300 dilutions were shown in orange and turquoise, respectively) and ACE2-Fc (3 $\mu \mathrm{g} / \mathrm{mL})$ (red) by bead-based ACE2-Bite capture assay. Wild-type 293 cell supernatant (Control supe, Blue) and staining buffer (None, Pink) were used as negative controls. (E) Binding of ACE2-Bite to Spike-GFP expressing T2 cell line and primary human T cells. HA staining of Spike-GFP expressing T2 cells (top panel) and CD8 T cells (bottom panel) when combined with ACE2-Bite (right plot) or control (Wild-type 293) (left plot) supernatant. (F) CD25 and GFP expression show activation and cytotoxicity state of resting CD8 T cells against Spike/GFP-expressing or control (transduced with GFP-expressing empty vector) 293 cells in the presence of ACE2-Bite or control supernatant. The experiments were replicated with similar results.

\section{Figure 4: Binding of ACE2-Bite to Spike proteins with different variant}

\section{mutations}

(A) Representation of ACE2-Bite binding to spike protein (wild-type or mutated) expressed on the cell surface membrane and its detection by immunostaining with an anti-HA antibody. Wild-type or mutant Spike proteins (Table 1) were expressed on 293 cells for ACE2-Bite/Spike binding 
assay. (B) Geometric mean intensity of anti-HA antibody staining used to detect ACE2-Bite molecules on mutant Spike-expressing cells. The cells were co-stained with an anti-Spike antibody and ACE2-Bite/anti-HA Tag antibody and analyzed via flow cytometry. For each condition, anti-Spike antibody-staining was used as a Spike protein marker and fluorescently equivalent gates were set before assessing the geometric mean fluorescent intensity of ACE2Bite-stained cells to determine the quantitative value of ACE2-Bite fluorescence intensity per Spike protein. Unpaired t test was used to determine the statistical significance. (C) CD25 expression of CD8 T cells when co-cultured with wild-type and mutated Spike protein plasmid transfected 293 cells (used in B) in the presence of ACE2-Bite supernatant.

\section{Figure 5: Binding of ACE2-Bite to SARS-CoV-2 Spike protein variants on}

\section{pseudotyped lentiviruses for virus neutralization}

(A) Schematic illustration of virus neutralization assay. ACE2-Bite and Spike (wild-type and mutated) pseudotyped lentiviruses are pre-incubated then added to ACE2-overexpressing 293 cells. (B) Representative FACS plots show neutralization data of the delta variant pseudotyped virus infection when pre-incubated with different concentrations of ACE2-Fc (top panel) or different dilutions of ACE2-Bite supernatant (bottom panel). The infection levels were determined 3 days later via flow cytometry based on GFP expression. (C) Line graph represents virus neutralization data of the lentiviruses pseudotyped with different Spike protein variants when preincubated with ACE2-Bite at different ACE2-Bite:virus ratios then added to ACE2-overexpressing 293s. These experiments were replicated twice with similar results. 


\section{Supplementary Figure 1: Selective cytotoxicity of ACE2 CAR and anti-}

\section{Spike CAR-T cells for spike expressing target cells.}

CAR engineered T cells cytotoxicity assays with Spike expressing and control 293 target cells. Control 293s were engineered with a GFP-expressing empty vector. Spike-expressing and control 293s were identified with GFP expression. Effector cells were identified by CD8 staining. T cell activation was determined via CD25 staining. CAR expressing T cells co-expressed RFP with CAR constructs.

\section{Supplementary Figure 2: Determining ACE2-Bite concentrations by a}

\section{capture assay.}

Area under the curve (AUC) values of ACE2-Bite molecules in supernatants from different conditions. Supernatants from ACE2-Bite secreting and wild-type suspension 293 cells were collected at several timepoints representing different cell densities ranging from 3 to $7 \mathrm{million} / \mathrm{mL}$. ACE2-Bite molecules taken from 3 million/mL cell culture supernatant were concentrated 5 -folds and 30-folds. Flow through supernatant from the concentration process (Filter flow through) and wild-type control supernatant were used as controls. Fluorescent beads coated with SpikeReceptor binding domain (S-RBD) were used to capture ACE2-Bite molecules in supernatants titrated from $1: 1$ to $1: 1000$ by 10 -fold serial dilutions were detected via a recombinant CD3-Fc fusion protein and an anti-Fc antibody. Geometric mean intensity of anti-Fc antibody fluorescence were used to generate curves which were used to calculate the area under the curve values. 


\section{Tables}

Table 1: Mutations in the Spike-Receptor Binding Domain

\begin{tabular}{|l|l|l|}
\hline Spike version & Note & Lineage \\
\hline Wild-type Spike & Wuhan strain S-RBD & Wuhan-Hu-1 \\
\hline E484K & In multiple variants & - \\
\hline N501Y & Alpha variant & B.1.1.7 \\
\hline E484K+ N501Y & In multiple variants & - \\
\hline E484K+ N501Y + K417N & Beta variant & B.1.351 \\
\hline L452R+T478K & Delta variant & B.1.617.2 \\
\hline L452R+T478K+K417N & Delta plus variant & B.1.617.2.1 \\
\hline
\end{tabular}

\section{Materials and Methods}

\section{ACE2 CAR construct}

CAR constructs consisting of CD8 alpha signal peptide, extracellular domain of ACE2 molecule or single chain variable fragment (scFv) of anti-CD19 or anti-Spike protein antibodies, CD8 hinge domain, CD8 transmembrane domain, 4-1BB (CD137) intracellular domain and CD3ろ domain were designed with Snapgene and synthesized via Genscript. ACE2 extracellular domain, CD8a signal peptide, CD8 hinge, CD8 transmembrane domain, 4-1BB intracellular domain and CD3 $\zeta$ domain sequences were obtained from Ensembl Gene Browser and codon optimized with SnapGene by removing the restriction enzyme recognition sites that are necessary for subsequent molecular cloning steps, while preserving the amino acid sequences. Anti-CD19 and 
anti-Spike scFv amino acid sequences were obtained from Addgene plasmids \#79125 and \#155364, respectively, reverse translated to DNA sequences and codon optimized with Snapgene 5.2.4. The constructs were then cloned into a lentiviral expression vector with a multiple cloning site separated from RFP reporter via an Internal Ribosomal Entry Site (IRES).

\section{Spike protein constructs}

Human codon optimized wild-type full-length SARS-CoV-2 Spike protein sequence was synthesized by MolecularCloud (MC_0101081) and then cloned into pLP/VSVG plasmid from Thermo Fisher under CMV promoter after removing the VSVG sequence via EcoRI-EcoRI restriction digestion. 5'-ACGACGGAATTCATGTTCGTCTTCCTGGTCCTG-3' and 5'ACGACGGAATTCTTAACAGCAGGAGCCACAGC-3' primers were used to generate wild-type SARS-CoV-2 Spike protein sequence without the Endoplasmic Reticulum Retention Signal (ERRS, last 19 amino acids of Spike) (Ou et al., 2020). E484K and N501Y mutated spike protein sequences without ERRS domain were obtained from VectorBuilder plasmids pRP[Exp]-CMVhuman beta globin intron>S(E484K,deltaC19)/3xFLAG and pRP[Exp]-CMV-human beta globin intron>S(N501Y,deltaC19)/3xFLAG, respectively. Since wild-type Spike protein did not have the FLAG tag and efficiently incorporated into the lentiviruses, FLAG Tags in each construct were removed to have the same amino acid sequences among all Spike constructs with the exception of the necessary mutations, via PCR amplification with 5'ACGACGGAATTCATGTTCGTTTTCCTTGTTCTGTTGC-3' ACGACGGAATTCTTAGCAACATGATCCGCAAGAGCA-3' primers and cloned into the same pLP expression plasmid. E484K+N501Y mutated, K417N+E484K+N501Y mutated (Beta variant, B.1.351, South African), L452R+T478K mutated (Delta variant, B.1.617.2) and K417N+L452R+T478K mutated (Delta plus variant, B.1.617.2.1) Spike protein sequences were built on top of E484K and N501Y mutated Spike protein sequences via overlap extension PCR 
using 2 new primers together with the 5' and 3' primers mentioned above for each single mutation insertion and cloned into the pLP expression plasmid. All mutation insertions were confirmed via Eton Bioscience DNA sequencing. Sequencing and overlap extension PCR primer sequences are available upon request. For stable wild-type Spike protein overexpression, wild-type Spike protein sequence without ERRS domain was cloned into a lentivector with a GFP marker under LTR promoter.

\section{VSVG and Spike Protein pseudotyped lentivirus production}

The lentiviruses pseudotyped with vesicular stomatitis virus G protein envelope were generated with HEK293T cells. Briefly, the lentivector plasmids containing the constructs were cotransfected with vesicular stomatitis virus G protein, pLP1, and pLP2 plasmids into HEK293T cells at $80-90 \%$ confluency using Lipofectamine 3000 (Invitrogen) according to the manufacturer's protocol. In the case of Spike protein pseudotyped lentiviruses, a lentivector plasmid containing GFP reporter was co-transfected with wild-type or mutated SARS-CoV-2 Spike protein plasmids in the same manner. The transfection medium was replaced with RPMI 1640 with 10\% FBS 6 hours post-transfection. Viral supernatants were collected 24 to 48 hours post-transfection and filtered through a $0.45-\mu m$ syringe filter (Millipore) to remove cellular debris. A Lenti-X concentrator (Takara Bio USA) was used according to the manufacturer's protocol to concentrate the virus $10-20 \times$ and the resulting lentiviral stocks were aliquoted and stored at $-80^{\circ} \mathrm{C}$. To measure viral titers of VSV-G pseudotyped lentiviruses, virus preparations were serially diluted on Jurkat cells and 3 days post-infection, infected cells were measured using flow cytometry and the number of cells transduced with $1 \mathrm{~mL}$ of virus supernatant was calculated as infectious units per milliliter. For spike protein pseudotyped lentiviruses, to measure viral titers, virus preparations were serially diluted on ACE2 over-expressing 293 cells, which were stained for their ACE2 expressions and confirmed \%100 positive. 72 hours after infection, GFP positive cells were 
counted using flow cytometry and the number of cells transduced with virus supernatant was calculated as infectious units/per mL. Based on these titer values, primary T cells, 293 T cells and T2 cells were transduced with a multiplicity of infection (MOI) of 3-10.

\section{ACE2-Bite design and production}

The ACE2-Bite construct consisting of ACE2 signal peptide, ACE2 extracellular domain, a linker peptide, an anti-CD3 antibody single-chain variable fragment, a His-Tag, and a Hemagglutinin (HA) Tag was designed with Snapgene and synthesized via Genscript. ACE2 signal peptide and extracellular domain sequences were obtained from Ensembl Gene Browser (Transcript ID: ENST00000252519.8). Anti-CD3 antibody single-chain variable fragment, His-Tag, and Hemagglutinin (HA) Tag sequences were obtained from Addgene plasmid \#85437. ACE2-Bite construct was cloned into an RFP marked lentivector under LTR promoter, and Expi293F TM suspension 293 cells from ThermoFisher were transduced with the ACE2-Bite expressing VSVG pseudotyped lentiviruses with multiplicity of infection of 5 . The cells were then grown in Expi293 ${ }^{\mathrm{TM}}$ Expression Medium in shaking flasks for 7 days until they reached maximum viable density. ACE2-Bite containing supernatant was then collected and filtered/concentrated up to 30 -fold with $30 \mathrm{kDa}$ MilliporeSigma ${ }^{\mathrm{TM}}$ Amicon $^{\mathrm{TM}}$ Ultra Centrifugal Filter Units. Concentrated ACE2-Bite and control supernatants were aliquoted and stored in $4^{\circ} \mathrm{C}$.

\section{Engineering CAR-T cells and Spike expressing target cells}

Healthy adult blood was obtained from AllCells. PBMCs were isolated using Ficoll-paque plus (GE Health care). CD8 T cells were purified using Dynal CD8 Positive Isolation Kit (from Invitrogen). CD8 T cells were $>99 \%$ pure and assessed by flow cytometry staining with CD8Pacific Blue antibody (Biolegend). Total CD8 T cells were activated using anti-CD3/CD28 coated beads (Invitrogen) at a 1:2 ratio (beads:cells) and infected with anti-CD19 CAR, anti-Spike CAR 
or ACE2 CAR VSVG pseudotyped lentiviral constructs with multiplicity of infection (MOI) of 5-10. The cells were then expanded in complete RPMI 1640 medium supplemented with 10\% Fetal Bovine Serum (FBS, Atlanta Biologicals), 1\% penicillin/streptomycin (Corning Cellgro) and $20 \mathrm{ng} / \mathrm{ml}$ of IL-2 and cultured at $37^{\circ} \mathrm{C}$ and $5 \% \mathrm{CO}_{2}$ supplemented incubators. Respective viruses were added 24 hours after the activation. Cells were expanded for 10-12 days and cytotoxicity assays were performed following their expansion. To generate HEK-293T cells that transiently expressed wild type and mutated spike protein (ATCC; mycoplasma-free low passage stock), the cells were transfected with Spike protein expressing pLP plasmids using Lipofectamine 3000 (Invitrogen) according to the manufacturer's protocol and stained for their spike protein expression 72 hours after the transfection as described in Staining and Flow cytometry Analysis. All engineered and wild-type HEK-293 and T2 cells were cultured in complete RPMI 1640 medium (RPMI 1640 supplemented with 10\% FBS; Atlanta Biologicals, Lawrenceville, GA), 8\% GlutaMAX (Life Technologies), 8\% sodium pyruvate, 8\% MEM vitamins, 8\% MEM nonessential amino acid, and $1 \%$ penicillin/streptomycin (all from Corning Cellgro). To generate T2s and 293s with stable Spike overexpression, wild-type T2 and 293 cells were transduced with $3 \mathrm{MOI}$ of Spike protein overexpressing VSVG lentivirus and proliferated. The infection levels were determined by GFP expression through Flow Cytometry analysis. For ACE2 overexpression in 293, wild-type ACE2 sequence was obtained from Ensembl Gene Browser (Transcript ID: ENST00000252519.8) and codon optimized with SnapGene by removing restriction enzyme recognition sites that are necessary for subsequent molecular cloning steps preserving the amino acid sequence, synthesized in GenScript and then cloned into a lentiviral vector. VSVG pseudotyped lentiviruses of respective constructs were generated as mentioned above and added to the cells with MOI of 3. Transduction levels were determined by ACE2 staining via Flow Cytometry 72 hours after the infection. ACE2 staining is described in Staining and flow cytometry analysis. 


\section{Flow cytometry analysis}

Cells were resuspended in staining buffer (PBS + $2 \%$ FBS) and incubated with fluorochromeconjugated antibodies for $30 \mathrm{~min}$ at $4^{\circ} \mathrm{C}$. CD8 $\mathrm{T}$ cells were identified with CD8-Pacific Blue antibody (Biolegend). Activation of CAR CD8 T cells was determined with CD25 staining using CD25-APC antibody (Biolegend). CAR expressions of ACE2 CAR and anti-Spike CAR and ACE2 expression of ACE2-293 cells were determined with SARS-CoV-2 S1 protein, Mouse IgG2a Fc Tag (Acro Biosystems) incubation followed with APC Goat anti-mouse IgG2a Fc Antibody (Invitrogen) staining and RFP expression. CAR expression of anti-CD19 CAR was determined with Human CD19 (20-291) Protein, Fc Tag, low endotoxin (Super affinity) (Acro) followed by a secondary staining with APC conjugated anti-human IgG Fc Antibody (Biolegend) and RFP expression. For cytotoxicity assay analysis, stably Spike protein-expressing T2 and 293 cell lines were identified with GFP marker. For Spike protein flow cytometry analysis, the cells were stained with Biotinylated Human ACE2 / ACEH Protein, Fc,Avitag (Acro Biosystems), then stained with APC anti-human IgG Fc Antibody clone HP6017 (Biolegend). Samples were acquired on a BD FACSymphony A5 analyzer and data were analyzed using FlowJo (BD Biosciences).

\section{Cytotoxicity assay}

Following the expansion of engineered CAR-T cells for 10-12 days, the cells were analyzed for their RFP and CAR expressions. Effector to target cell ratio was calculated based on the number of CAR expressing cells. CAR expressing cells were titrated from 2:1 to 1:16 effector to target cell ratio at 2-fold dilutions while the target cell number was constant. For ACE2-Bite cytotoxicity assays, resting total CD8 T cells were combined with wild-type Spike overexpressing 293 cells, empty vector transduced 293 cells, mutated Spike protein transfected 293 cells and wild-type 293 cells in a 4:1 Effector/Target cell ratio, and ACE2-Bite and control supernatant were added in 1:10 supernatant/cell medium ratio. Cytotoxicity assay conditions were analyzed with Flow Cytometry 
at 72 hours of co-incubation and the cells were identified as described in Staining and flow cytometry analysis.

\section{ACE2-Bite detection assay}

Supernatants from ACE2-Bite secreting and wild-type suspension 293 cells were collected at several timepoints with different cell densities ranging from 3 to 7 million $/ \mathrm{mL}$. ACE2-Bite molecules taken from 3 million/mL cell culture supernatant were concentrated 5 -folds and 30 folds by using $15 \mathrm{~mL} 30 \mathrm{kDa}$ MilliporeSigma ${ }^{\mathrm{TM}}$ Amicon $^{\mathrm{TM}}$ Ultra Centrifugal Filter Units. To capture the ACE2-Bite or ACE2-Fc molecules, The DevScreen SAv Bead kit (Essen BioScience, MI) was used. Biotinylated 2019-nCoV (COVID-19) spike protein RBD, His, Avitag was coated to SAv Beads according to manufacturer's instructions. Confirmation of successful bead conjugation was determined by staining with anti-His Tag (Biolegend) and flow cytometry analysis. S-RBD conjugated beads were then used as capture beads in flow immunoassay where they were incubated with recombinant Human ACE2-Fc (Acro Biosystems) or ACE2-Bite supernatant samples for $1 \mathrm{~h}$ at room temperature. Supernatant samples were assayed at a $1: 1$ starting dilution and three additional tenfold serial dilutions. ACE2-Fc was tested at a $30 \mu \mathrm{g} / \mathrm{mL}$ starting concentration and in additional five threefold serial dilutions. Detection reagent was prepared using Human CD3 epsilon Protein, Mouse IgG2a Fc Tag (Acro) and Phycoerythrin-conjugated Goat anti-Mouse IgG2a Cross-Adsorbed Secondary Antibody (Fisher) for ACE2-Bite and APC anti-human IgG Fc Antibody clone HP6017 (Biolegend) for ACE2-Fc were added to the wells and incubated for another hour at room temperature. Plates were then washed twice with PBS and analyzed by flow cytometry using iQue Screener Plus (IntelliCyt, MI). Flow cytometry data were analyzed using FlowJo (BD biosciences). DevScreen SAv Beads were gated using FSC-H/SSC$\mathrm{H}$, and singlet beads gate was created using FSC-A/FSC-H. Gates for different DevScreen SAv Beads were determined based on their fluorescence signature on $R L 1-H / R L 2-H$ plot (on iQue 
plus). PE fluorescence median, which is directly associated with each single plex beads was determined using BL2-H (on iQue plus). Geometric means of PE fluorescence in different titrations were used to generate the titration curve and the area under the curve was calculated using GraphPad Prism 9.0 software (GraphPad Software).

\section{Spike pseudotyped virus neutralization assay}

Three-fold serially diluted recombinant human ACE2-Fc (Acro Biosystems) or two-fold serially diluted ACE2-Bite and control supernatants were incubated with GFP-encoding SARS-CoV-2 Spike pseudotyped viruses with 0.2 multiplicity of infection (MOI) for 1 hour at $37^{\circ} \mathrm{C}$ degrees. The mixtures were subsequently incubated with ACE2+ 293 cells, which were previously stained for their ACE2 expressions and confirmed $\% 100$ positive before neutralization assays, for $72 \mathrm{~h}$ hours after which cells were collected, washed with FACS buffer (1xPBS+2\% FBS) and analyzed by flow cytometry using BD FACSymphony A5 analyzer. Cells that do not express GFP were used to define the boundaries between non-infected and infected cell populations. Percent infection was normalized for samples derived from cells infected with SARS-CoV-2 pseudotyped virus in the absence of ACE2-Fc or ACE2-Bite.

\section{Statistical Analyses and Reproducibility}

All statistical analyses were performed and graphs were prepared using GraphPad Prism V9 software. The numbers of repeats for each experiment were described in the associated figure legends. 
bioRxiv preprint doi: https://doi.org/10.1101/2022.01.19.476940; this version posted January 20,2022. The copyright holder for this preprint

(which was not certified by peer review) is the author/funder, who has granted bioRxiv a license to display the preprint in perpetuity. It is made available under aCC-BY-NC-ND 4.0 International license.

\section{Data and Material Availability}

The source data for the Figures along with the Supplementary Figures are available upon request.

All unique/stable reagents generated in this study are available from the lead contact with a completed materials transfer agreement 


\section{References}

Ahmad, A., Uddin, S., and Steinhoff, M. (2020). CAR-T Cell Therapies: An Overview of Clinical Studies Supporting Their Approved Use against Acute Lymphoblastic Leukemia and Large BCell Lymphomas. Int J Mol Sci 21. 10.3390/ijms21113906.

Bailey, J.R., Barnes, E., and Cox, A.L. (2019). Approaches, Progress, and Challenges to Hepatitis C Vaccine Development. Gastroenterology 156, 418-430.

10.1053/j.gastro.2018.08.060.

Barton, M.I., MacGowan, S.A., Kutuzov, M.A., Dushek, O., Barton, G.J., and van der Merwe, P.A. (2021). Effects of common mutations in the SARS-CoV-2 Spike RBD and its ligand, the human ACE2 receptor on binding affinity and kinetics. Elife 10. 10.7554/eLife.70658.

Baum, A., Ajithdoss, D., Copin, R., Zhou, A., Lanza, K., Negron, N., Ni, M., Wei, Y., Mohammadi, K., Musser, B., et al. (2020). REGN-COV2 antibodies prevent and treat SARSCoV-2 infection in rhesus macaques and hamsters. Science 370, 1110-1115.

10.1126/science.abe2402.

Brey, C.U., Proff, J., Teufert, N., Salzer, B., Brozy, J., Munz, M., Pendzialek, J., Ensser, A., Holter, W., and Lehner, M. (2018). A gB/CD3 bispecific BiTE antibody construct for targeting Human Cytomegalovirus-infected cells. Sci Rep 8, 17453. 10.1038/s41598-018-36055-2.

Case, J.B., Rothlauf, P.W., Chen, R.E., Liu, Z., Zhao, H., Kim, A.S., Bloyet, L.M., Zeng, Q., Tahan, S., Droit, L., et al. (2020). Neutralizing Antibody and Soluble ACE2 Inhibition of a Replication-Competent VSV-SARS-CoV-2 and a Clinical Isolate of SARS-CoV-2. Cell Host Microbe 28, 475-485 e475. 10.1016/j.chom.2020.06.021. 
Cattin-Ortola, J., Welch, L.G., Maslen, S.L., Papa, G., James, L.C., and Munro, S. (2021).

Sequences in the cytoplasmic tail of SARS-CoV-2 Spike facilitate expression at the cell surface and syncytia formation. Nat Commun 12, 5333. 10.1038/s41467-021-25589-1.

Cellina, M., Orsi, M., Bombaci, F., Sala, M., Marino, P., and Oliva, G. (2020). Favorable changes of CT findings in a patient with COVID-19 pneumonia after treatment with tocilizumab. Diagn Interv Imaging 101, 323-324. 10.1016/j.diii.2020.03.010.

Depil, S., Duchateau, P., Grupp, S.A., Mufti, G., and Poirot, L. (2020). 'Off-the-shelf' allogeneic CAR T cells: development and challenges. Nature Reviews Drug Discovery 19, 185-199.

10.1038/s41573-019-0051-2.

Dieterle, M.E., Haslwanter, D., Bortz, R.H., 3rd, Wirchnianski, A.S., Lasso, G., Vergnolle, O., Abbasi, S.A., Fels, J.M., Laudermilch, E., Florez, C., et al. (2020). A Replication-Competent Vesicular Stomatitis Virus for Studies of SARS-CoV-2 Spike-Mediated Cell Entry and Its Inhibition. Cell Host Microbe 28, 486-496 e486. 10.1016/j.chom.2020.06.020.

Dogan, M., Kozhaya, L., Placek, L., Gunter, C., Yigit, M., Hardy, R., Plassmeyer, M., Coatney, P., Lillard, K., Bukhari, Z., et al. (2021). SARS-CoV-2 specific antibody and neutralization assays reveal the wide range of the humoral immune response to virus. Commun Biol 4, 129 . 10.1038/s42003-021-01649-6.

Duan, L., Zheng, Q., Zhang, H., Niu, Y., Lou, Y., and Wang, H. (2020). The SARS-CoV-2 Spike Glycoprotein Biosynthesis, Structure, Function, and Antigenicity: Implications for the Design of Spike-Based Vaccine Immunogens. Front Immunol 11, 576622. 10.3389/fimmu.2020.576622.

Ferrari, F., Bellone, S., Black, J., Schwab, C.L., Lopez, S., Cocco, E., Bonazzoli, E., Predolini, F., Menderes, G., Litkouhi, B., et al. (2015). Solitomab, an EpCAM/CD3 bispecific antibody 
construct $(\mathrm{BiTE}(\mathrm{R}))$, is highly active against primary uterine and ovarian carcinosarcoma cell lines in vitro. J Exp Clin Cancer Res 34, 123. 10.1186/s13046-015-0241-7.

Full, F., Lehner, M., Thonn, V., Goetz, G., Scholz, B., Kaufmann, K.B., Mach, M., Abken, H., Holter, W., and Ensser, A. (2010). T cells engineered with a cytomegalovirus-specific chimeric immunoreceptor. J Virol 84, 4083-4088. 10.1128/JVI.02117-09.

Gandhi, R.T., Lynch, J.B., and Del Rio, C. (2020). Mild or Moderate Covid-19. N Engl J Med 383, 1757-1766. 10.1056/NEJMcp2009249.

Glasgow, A., Glasgow, J., Limonta, D., Solomon, P., Lui, I., Zhang, Y., Nix, M.A., Rettko, N.J., Lim, S.A., Zha, S., et al. (2020). Engineered ACE2 receptor traps potently neutralize SARSCoV-2. bioRxiv. 10.1101/2020.07.31.231746.

Goldstein, R.L., Goyos, A., Li, C.M., Deegen, P., Bogner, P., Sternjak, A., Thomas, O., Klinger, M., Wahl, J., Friedrich, M., et al. (2020). AMG 701 induces cytotoxicity of multiple myeloma cells and depletes plasma cells in cynomolgus monkeys. Blood Adv 4, 4180-4194.

10.1182/bloodadvances.2020002565.

Gottlieb, R.L., Nirula, A., Chen, P., Boscia, J., Heller, B., Morris, J., Huhn, G., Cardona, J., Mocherla, B., Stosor, V., et al. (2021). Effect of Bamlanivimab as Monotherapy or in Combination With Etesevimab on Viral Load in Patients With Mild to Moderate COVID-19: A Randomized Clinical Trial. JAMA 325, 632-644. 10.1001/jama.2021.0202.

Group, R.C., Horby, P., Lim, W.S., Emberson, J.R., Mafham, M., Bell, J.L., Linsell, L., Staplin, N., Brightling, C., Ustianowski, A., et al. (2021). Dexamethasone in Hospitalized Patients with Covid-19. N Engl J Med 384, 693-704. 10.1056/NEJMoa2021436. 
Guimaraes, P.O., Quirk, D., Furtado, R.H., Maia, L.N., Saraiva, J.F., Antunes, M.O., Kalil Filho, R., Junior, V.M., Soeiro, A.M., Tognon, A.P., et al. (2021). Tofacitinib in Patients Hospitalized with Covid-19 Pneumonia. N Engl J Med 385, 406-415. 10.1056/NEJMoa2101643.

Harvey, W.T., Carabelli, A.M., Jackson, B., Gupta, R.K., Thomson, E.C., Harrison, E.M., Ludden, C., Reeve, R., Rambaut, A., Peacock, S.J., et al. (2021). SARS-CoV-2 variants, spike mutations and immune escape. Nature Reviews Microbiology 19, 409-424. 10.1038/s41579021-00573-0.

Haschke, M., Schuster, M., Poglitsch, M., Loibner, H., Salzberg, M., Bruggisser, M., Penninger, J., and Krahenbuhl, S. (2013). Pharmacokinetics and pharmacodynamics of recombinant human angiotensin-converting enzyme 2 in healthy human subjects. Clin Pharmacokinet 52, 783-792. 10.1007/s40262-013-0072-7.

Hoffmann, M., Kleine-Weber, H., Schroeder, S., Kruger, N., Herrler, T., Erichsen, S., Schiergens, T.S., Herrler, G., Wu, N.H., Nitsche, A., et al. (2020). SARS-CoV-2 Cell Entry Depends on ACE2 and TMPRSS2 and Is Blocked by a Clinically Proven Protease Inhibitor. Cell 181, 271-280 e278. 10.1016/j.cell.2020.02.052.

Horn, L.A., Ciavattone, N.G., Atkinson, R., Woldergerima, N., Wolf, J., Clements, V.K., Sinha, P., Poudel, M., and Ostrand-Rosenberg, S. (2017). CD3xPDL1 bi-specific T cell engager (BiTE) simultaneously activates T cells and NKT cells, kills PDL1(+) tumor cells, and extends the survival of tumor-bearing humanized mice. Oncotarget 8, 57964-57980.

10.18632/oncotarget.19865.

Hosseini, I., Gadkar, K., Stefanich, E., Li, C.C., Sun, L.L., Chu, Y.W., and Ramanujan, S. (2020). Mitigating the risk of cytokine release syndrome in a Phase I trial of CD20/CD3 
bispecific antibody mosunetuzumab in NHL: impact of translational system modeling. NPJ Syst Biol Appl 6, 28. 10.1038/s41540-020-00145-7.

Huet, T., Beaussier, H., Voisin, O., Jouveshomme, S., Dauriat, G., Lazareth, I., Sacco, E., Naccache, J.M., Bezie, Y., Laplanche, S., et al. (2020). Anakinra for severe forms of COVID-19: a cohort study. Lancet Rheumatol 2, e393-e400. 10.1016/S2665-9913(20)30164-8.

Johnston, M.I., and Fauci, A.S. (2008). An HIV vaccine--challenges and prospects. N Engl J Med 359, 888-890. 10.1056/NEJMp0806162.

Kantarjian, H., Stein, A., Gokbuget, N., Fielding, A.K., Schuh, A.C., Ribera, J.M., Wei, A., Dombret, H., Foa, R., Bassan, R., et al. (2017). Blinatumomab versus Chemotherapy for Advanced Acute Lymphoblastic Leukemia. N Engl J Med 376, 836-847.

10.1056/NEJMoa1609783.

Khan, A., Benthin, C., Zeno, B., Albertson, T.E., Boyd, J., Christie, J.D., Hall, R., Poirier, G., Ronco, J.J., Tidswell, M., et al. (2017). A pilot clinical trial of recombinant human angiotensinconverting enzyme 2 in acute respiratory distress syndrome. Crit Care 21, 234.

10.1186/s13054-017-1823-x.

Kruse, R.L., Shum, T., Tashiro, H., Barzi, M., Yi, Z., Whitten-Bauer, C., Legras, X., BissigChoisat, B., Garaigorta, U., Gottschalk, S., and Bissig, K.D. (2018). HBsAg-redirected T cells exhibit antiviral activity in HBV-infected human liver chimeric mice. Cytotherapy 20, 697-705. 10.1016/j.jcyt.2018.02.002.

Kumaresan, P.R., Manuri, P.R., Albert, N.D., Maiti, S., Singh, H., Mi, T., Roszik, J., Rabinovich, B., Olivares, S., Krishnamurthy, J., et al. (2014). Bioengineering T cells to target carbohydrate to treat opportunistic fungal infection. Proc Natl Acad Sci U S A 111, 10660-10665.

10.1073/pnas.1312789111. 
Laffeber, C., de Koning, K., Kanaar, R., and Lebbink, J.H.G. (2021). Experimental Evidence for Enhanced Receptor Binding by Rapidly Spreading SARS-CoV-2 Variants. J Mol Biol 433, 167058. 10.1016/j.jmb.2021.167058.

Leibman, R.S., Richardson, M.W., Ellebrecht, C.T., Maldini, C.R., Glover, J.A., Secreto, A.J., Kulikovskaya, I., Lacey, S.F., Akkina, S.R., Yi, Y., et al. (2017). Supraphysiologic control over HIV-1 replication mediated by CD8 T cells expressing a re-engineered CD4-based chimeric antigen receptor. PLoS Pathog 13, e1006613. 10.1371/journal.ppat.1006613.

Lutterbuese, R., Raum, T., Kischel, R., Hoffmann, P., Mangold, S., Rattel, B., Friedrich, M., Thomas, O., Lorenczewski, G., Rau, D., et al. (2010). T cell-engaging BiTE antibodies specific for EGFR potently eliminate KRAS- and BRAF-mutated colorectal cancer cells. Proc Natl Acad Sci U S A 107, 12605-12610. 10.1073/pnas.1000976107.

Maldini, C.R., Ellis, G.I., and Riley, J.L. (2018). CAR T cells for infection, autoimmunity and allotransplantation. Nat Rev Immunol 18, 605-616. 10.1038/s41577-018-0042-2.

Marconi, V.C., Ramanan, A.V., de Bono, S., Kartman, C.E., Krishnan, V., Liao, R., Piruzeli, M.L.B., Goldman, J.D., Alatorre-Alexander, J., de Cassia Pellegrini, R., et al. (2021). Efficacy and safety of baricitinib for the treatment of hospitalised adults with COVID-19 (COVBARRIER): a randomised, double-blind, parallel-group, placebo-controlled phase 3 trial. Lancet Respir Med. 10.1016/S2213-2600(21)00331-3.

Mitsuyasu, R.T., Anton, P.A., Deeks, S.G., Scadden, D.T., Connick, E., Downs, M.T., Bakker, A., Roberts, M.R., June, C.H., Jalali, S., et al. (2000). Prolonged survival and tissue trafficking following adoptive transfer of CD4zeta gene-modified autologous CD4(+) and CD8(+) T cells in human immunodeficiency virus-infected subjects. Blood 96, 785-793. 
Monk, P.D., Marsden, R.J., Tear, V.J., Brookes, J., Batten, T.N., Mankowski, M., Gabbay, F.J., Davies, D.E., Holgate, S.T., Ho, L.P., et al. (2021). Safety and efficacy of inhaled nebulised interferon beta-1a (SNG001) for treatment of SARS-CoV-2 infection: a randomised, doubleblind, placebo-controlled, phase 2 trial. Lancet Respir Med 9, 196-206. 10.1016/S2213$2600(20) 30511-7$.

Monteil, V., Kwon, H., Prado, P., Hagelkruys, A., Wimmer, R.A., Stahl, M., Leopoldi, A., Garreta, E., Hurtado Del Pozo, C., Prosper, F., et al. (2020). Inhibition of SARS-CoV-2 Infections in Engineered Human Tissues Using Clinical-Grade Soluble Human ACE2. Cell 181, 905-913 e907. 10.1016/j.cell.2020.04.004.

Ou, X., Liu, Y., Lei, X., Li, P., Mi, D., Ren, L., Guo, L., Guo, R., Chen, T., Hu, J., et al. (2020). Characterization of spike glycoprotein of SARS-CoV-2 on virus entry and its immune crossreactivity with SARS-CoV. Nat Commun 11, 1620. 10.1038/s41467-020-15562-9.

Sautto, G.A., Wisskirchen, K., Clementi, N., Castelli, M., Diotti, R.A., Graf, J., Clementi, M., Burioni, R., Protzer, U., and Mancini, N. (2016). Chimeric antigen receptor (CAR)-engineered T cells redirected against hepatitis C virus (HCV) E2 glycoprotein. Gut 65, 512-523.

10.1136/gutjnl-2014-308316.

Savoldo, B., Ramos, C.A., Liu, E., Mims, M.P., Keating, M.J., Carrum, G., Kamble, R.T., Bollard, C.M., Gee, A.P., Mei, Z., et al. (2011). CD28 costimulation improves expansion and persistence of chimeric antigen receptor-modified T cells in lymphoma patients. J Clin Invest $121,1822-$ 1826. 10.1172/JCl46110.

Silhol, F., Sarlon, G., Deharo, J.C., and Vaisse, B. (2020). Downregulation of ACE2 induces overstimulation of the renin-angiotensin system in COVID-19: should we block the reninangiotensin system? Hypertens Res 43, 854-856. 10.1038/s41440-020-0476-3. 
Sterner, R.C., and Sterner, R.M. (2021). CAR-T cell therapy: current limitations and potential strategies. Blood Cancer J 11, 69. 10.1038/s41408-021-00459-7.

Subklewe, M. (2021). BiTEs better than CAR T cells. Blood Adv 5, 607-612.

10.1182/bloodadvances.2020001792.

Sung, J.A., Pickeral, J., Liu, L., Stanfield-Oakley, S.A., Lam, C.Y., Garrido, C., Pollara, J., LaBranche, C., Bonsignori, M., Moody, M.A., et al. (2015). Dual-Affinity Re-Targeting proteins direct T cell-mediated cytolysis of latently HIV-infected cells. J Clin Invest $125,4077-4090$. $10.1172 / \mathrm{JCl} 82314$.

Tang, X., Zhou, Y., Li, W., Tang, Q., Chen, R., Zhu, J., and Feng, Z. (2014). T cells expressing a LMP1-specific chimeric antigen receptor mediate antitumor effects against LMP1-positive nasopharyngeal carcinoma cells in vitro and in vivo. J Biomed Res 28, 468-475.

10.7555/JBR.28.20140066.

Wan, Q., Kozhaya, L., Imberg, K., Mercer, F., Zhong, S., Krogsgaard, M., and Unutmaz, D. (2013). Probing the effector and suppressive functions of human T cell subsets using antigenspecific engineered T cell receptors. PLoS One 8, e56302. 10.1371/journal.pone.0056302.

Wraith, D.C. (2017). The Future of Immunotherapy: A 20-Year Perspective. Front Immunol 8, 1668. 10.3389/fimmu.2017.01668.

Wu, Q., Dudley, M.Z., Chen, X., Bai, X., Dong, K., Zhuang, T., Salmon, D., and Yu, H. (2021). Evaluation of the safety profile of COVID-19 vaccines: a rapid review. BMC Med 19, 173. 10.1186/s12916-021-02059-5. 
Yu, S., Zhang, J., Yan, Y., Yao, X., Fang, L., Xiong, H., Liu, Y., Chu, Q., Zhou, P., and Wu, K. (2019). A novel asymmetrical anti-HER2/CD3 bispecific antibody exhibits potent cytotoxicity for HER2-positive tumor cells. J Exp Clin Cancer Res 38, 355. 10.1186/s13046-019-1354-1.

Zoufaly, A., Poglitsch, M., Aberle, J.H., Hoepler, W., Seitz, T., Traugott, M., Grieb, A., Pawelka, E., Laferl, H., Wenisch, C., et al. (2020). Human recombinant soluble ACE2 in severe COVID19. Lancet Respir Med 8, 1154-1158. 10.1016/S2213-2600(20)30418-5. 
Figure 1

A

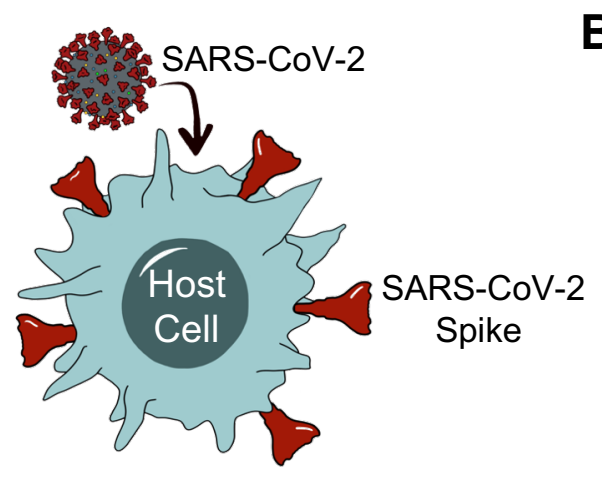

B

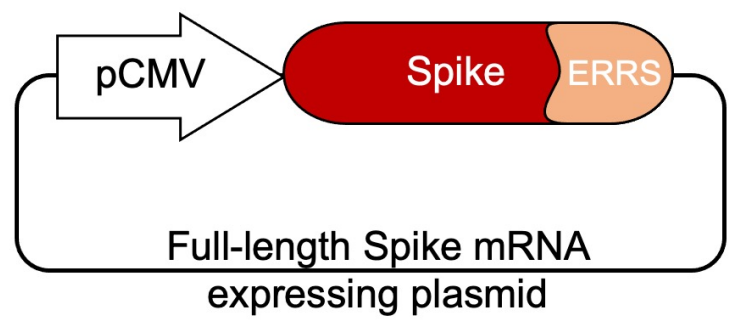

C

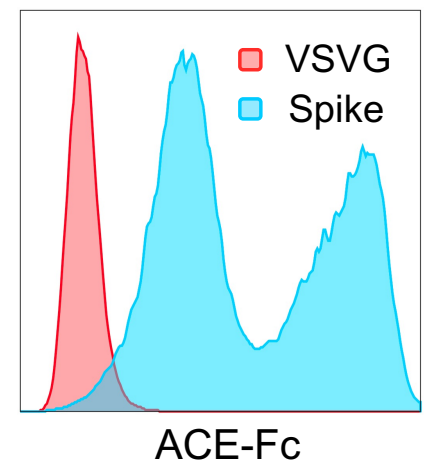

E

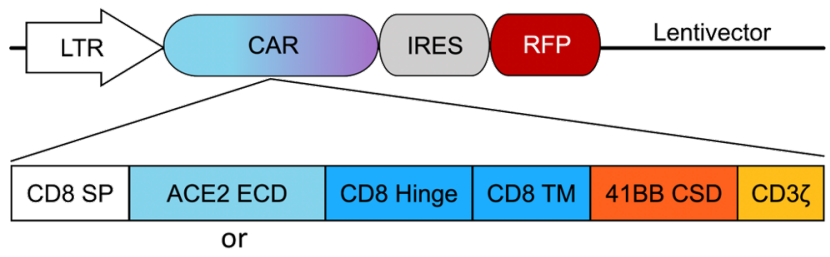

Anti-Spike ScFv

Lentiviral transduction
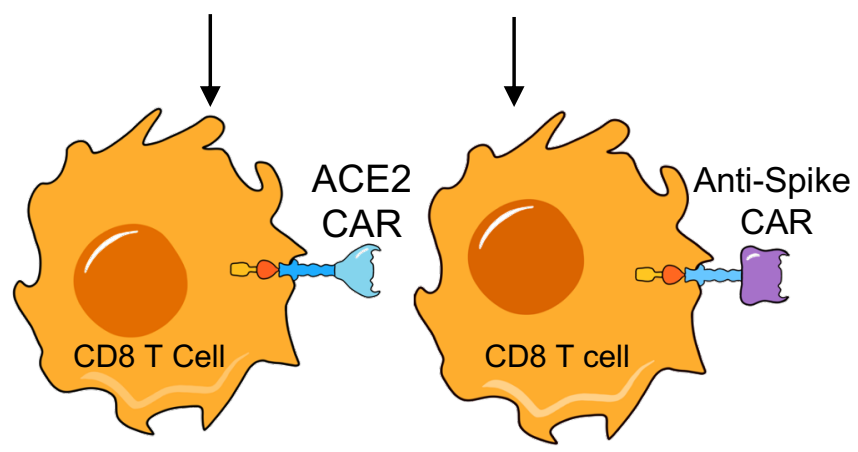

D Empty Lentivector Spike-GFP Lentivector
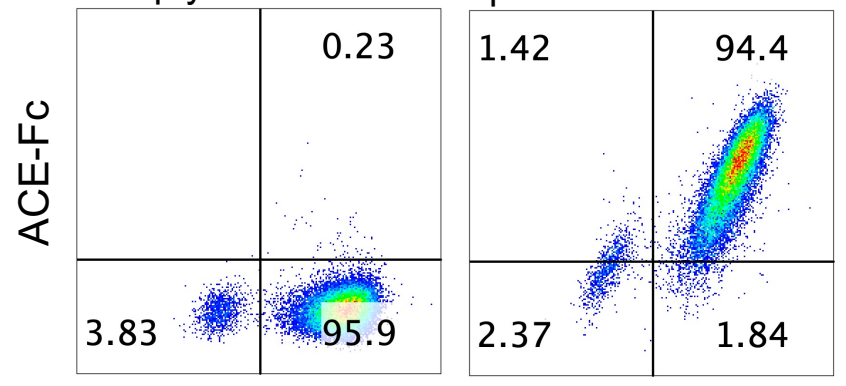

GFP

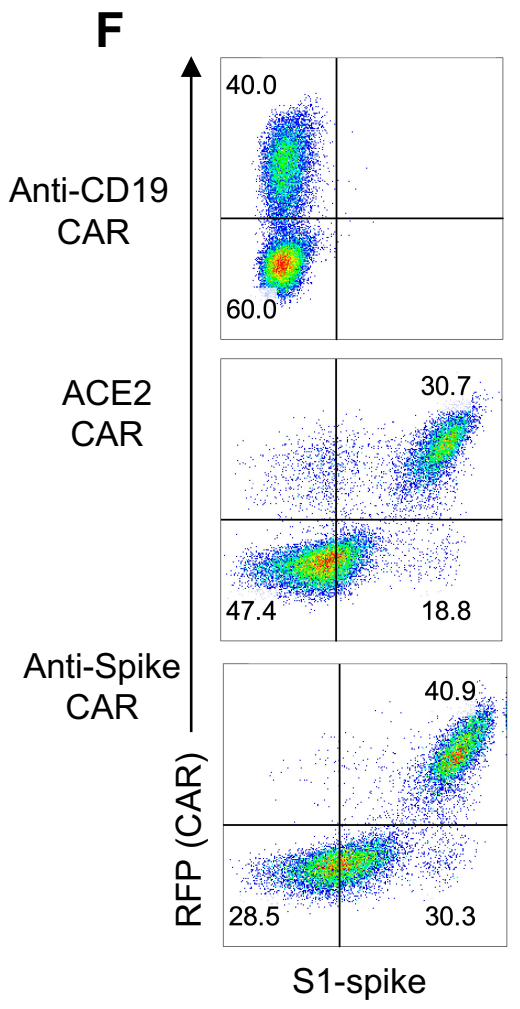


Figure 2

A

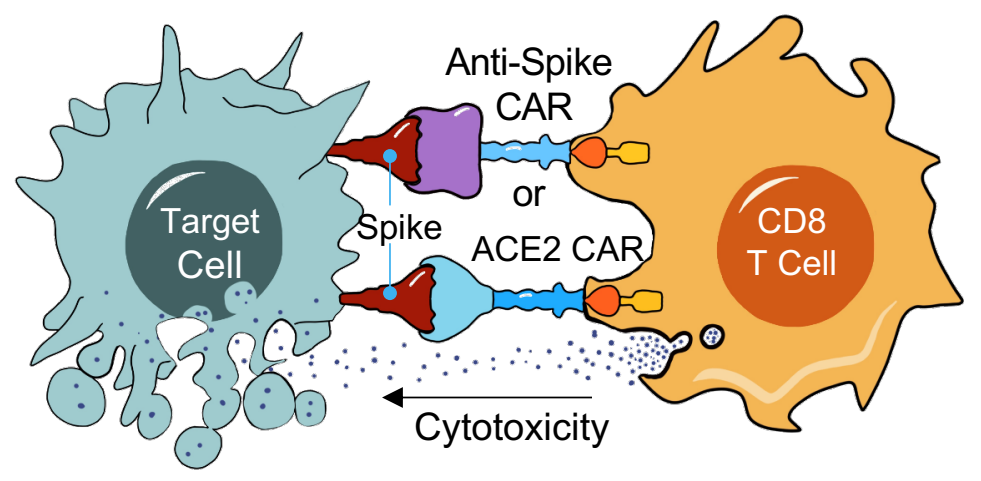

B

Anti-CD19 CAR (control)

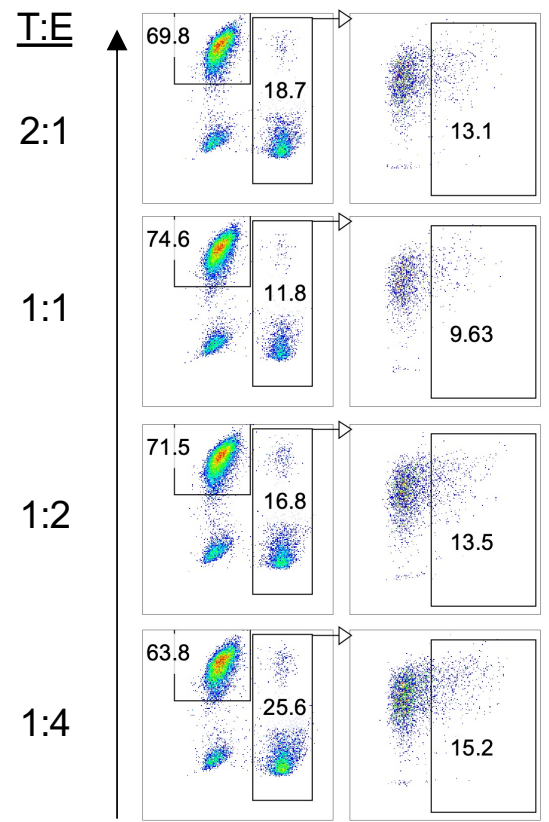

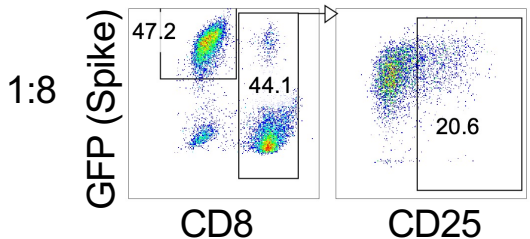

ACE2 CAR
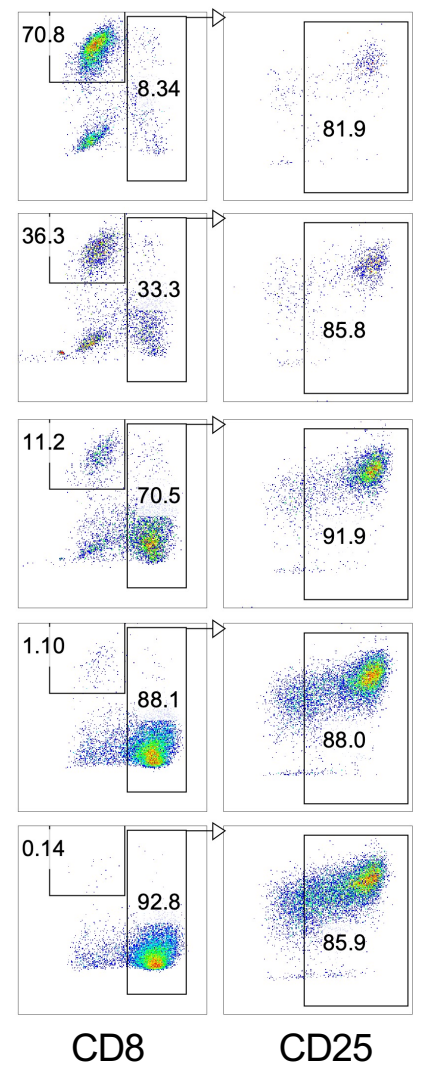

Anti-Spike CAR
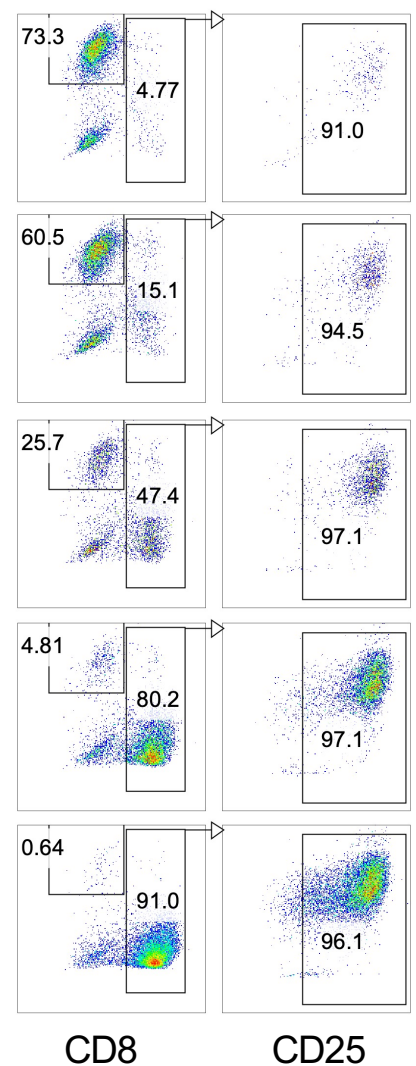

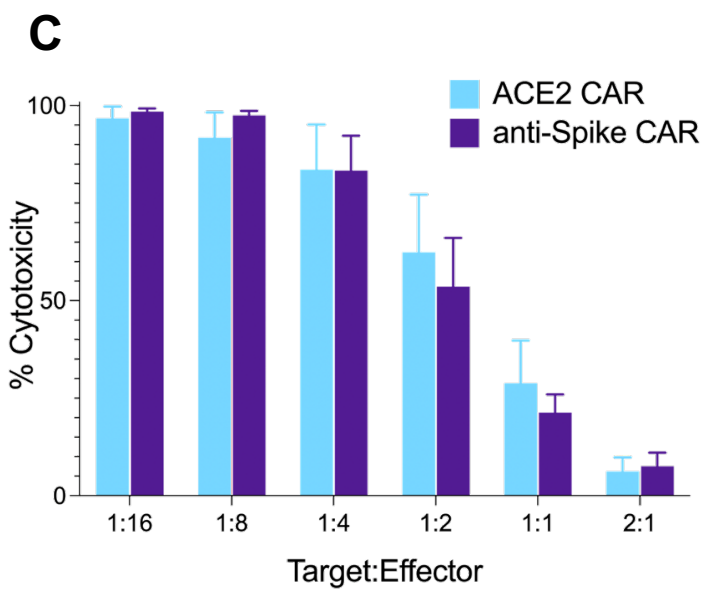

D

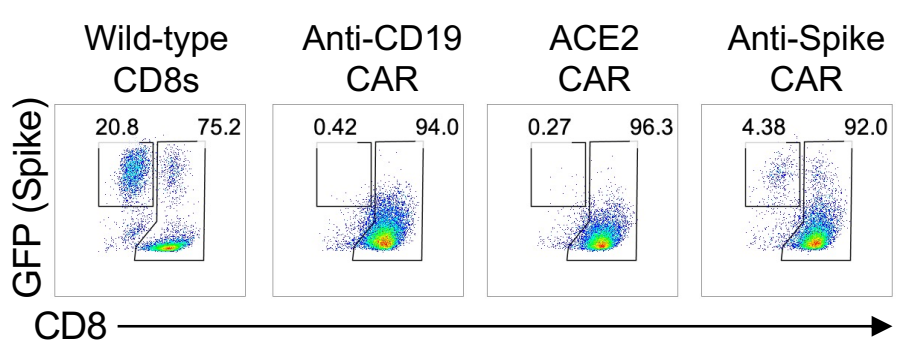


Figure 3
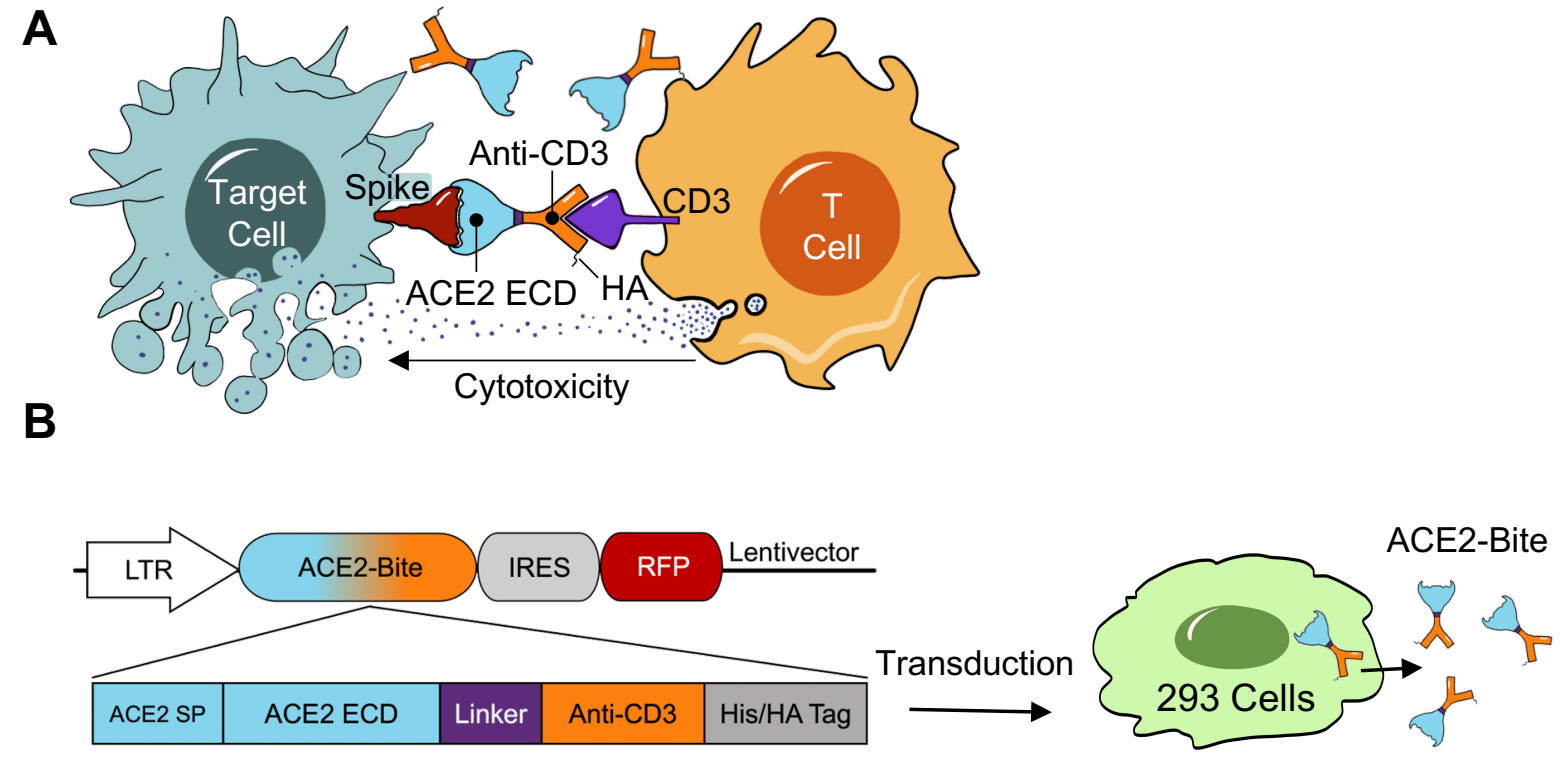

C

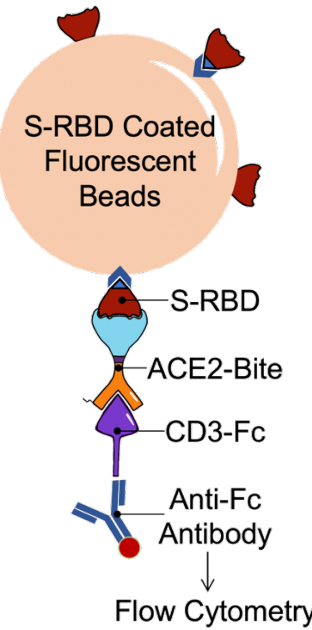

D
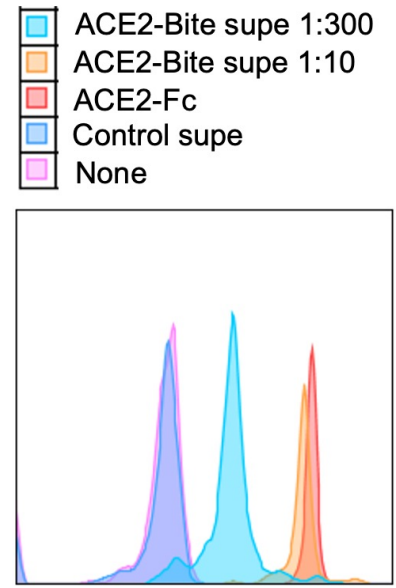

E

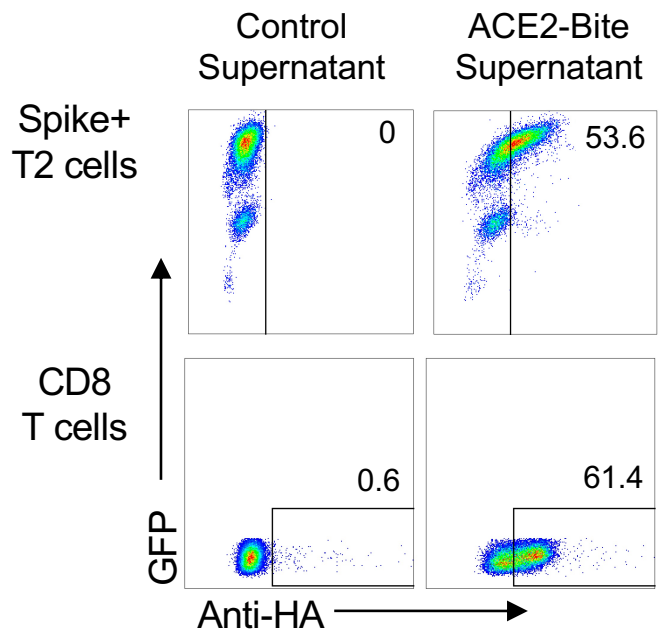

F Control 293 cells

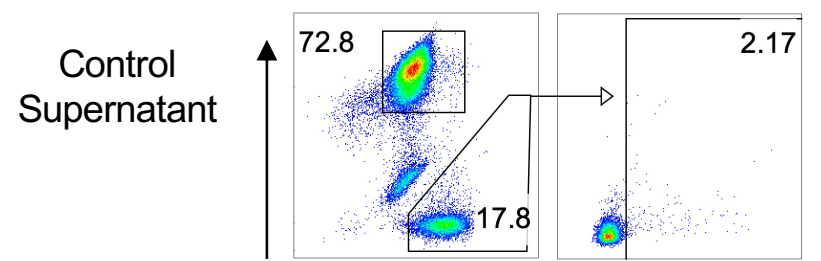

ACE2-Bite Supernatant

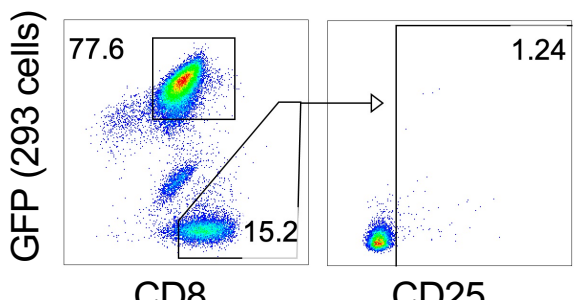

CD8
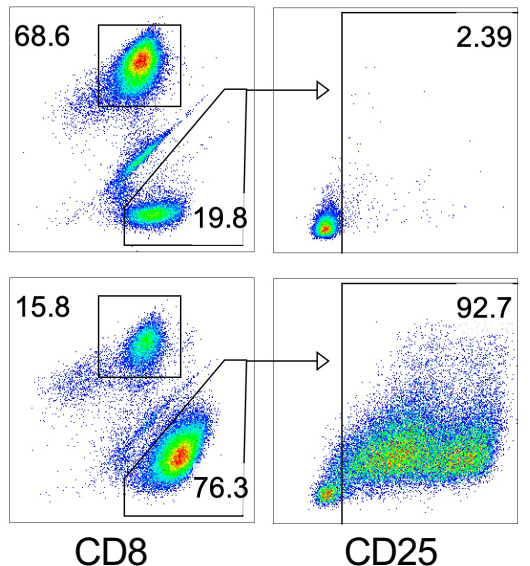
Figure 4

A

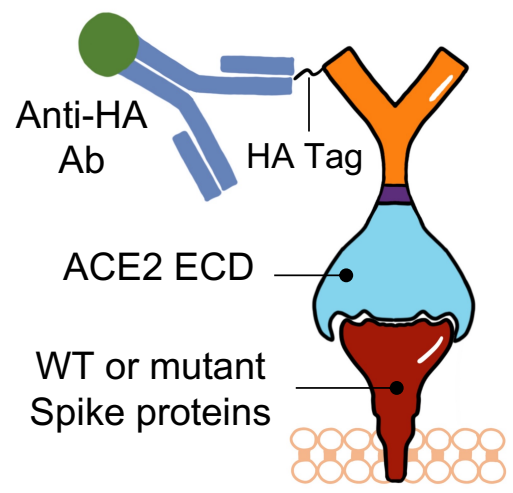

Cell membrane

B
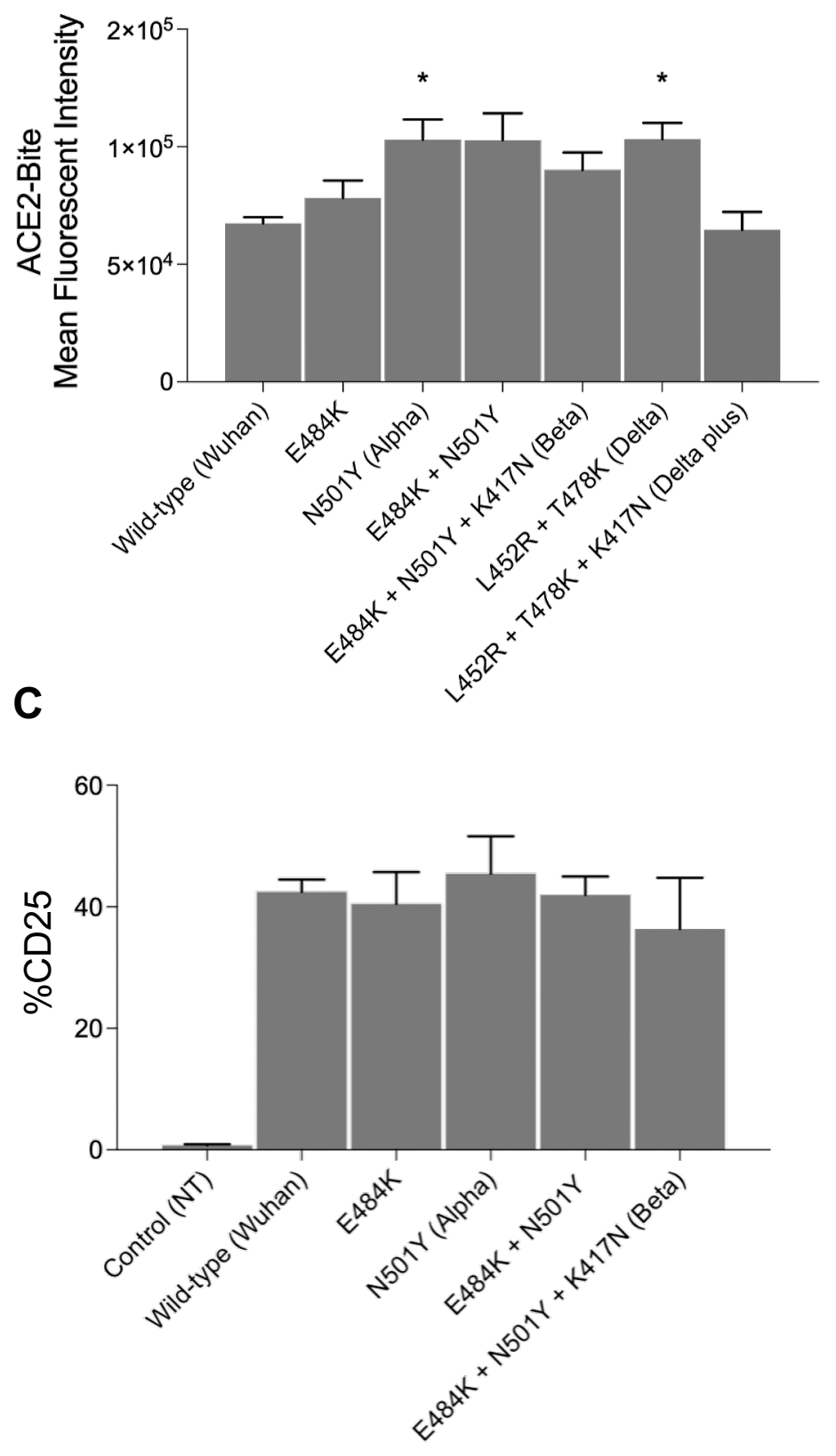
Figure 5

A

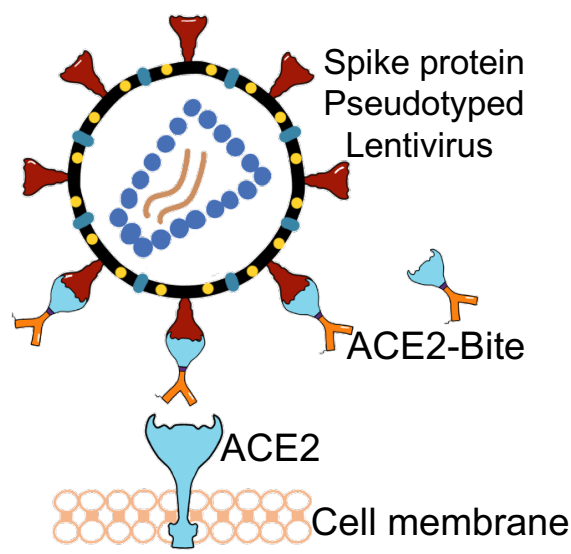

B
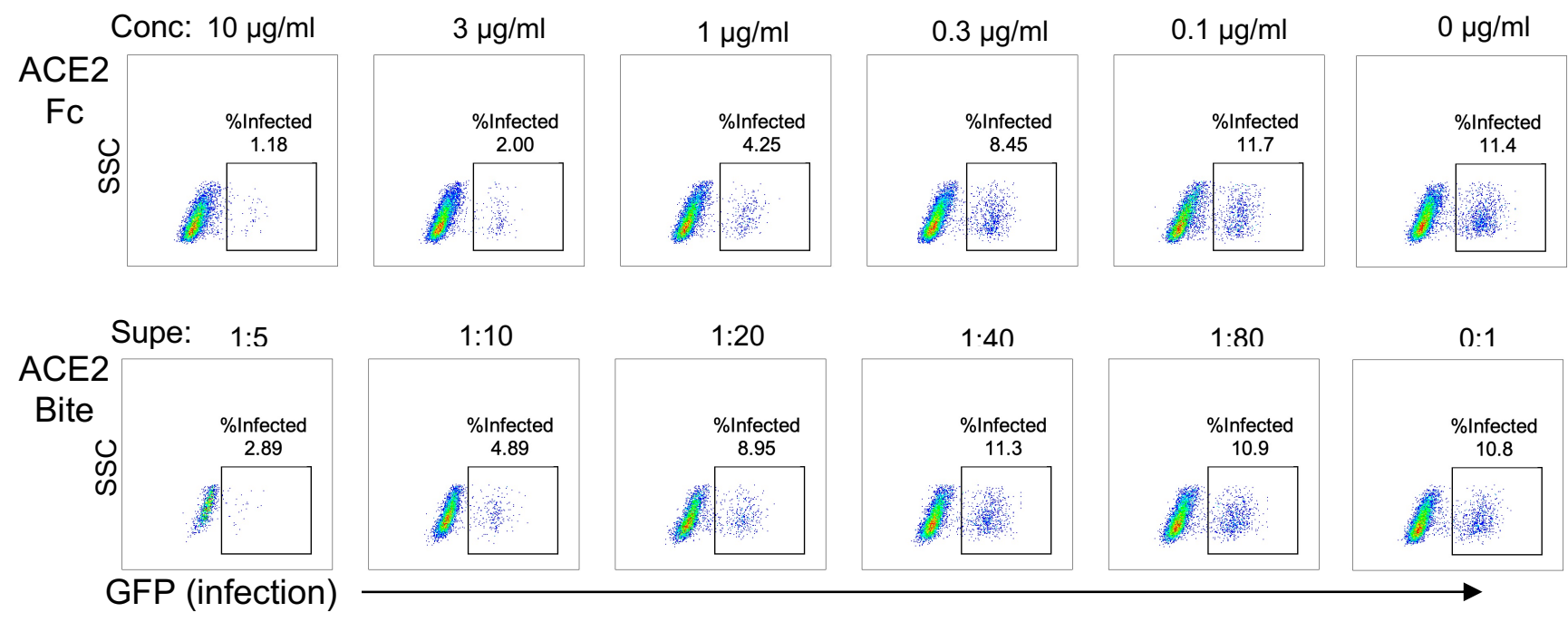

0:1

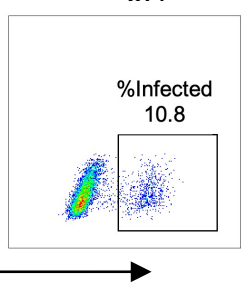

C

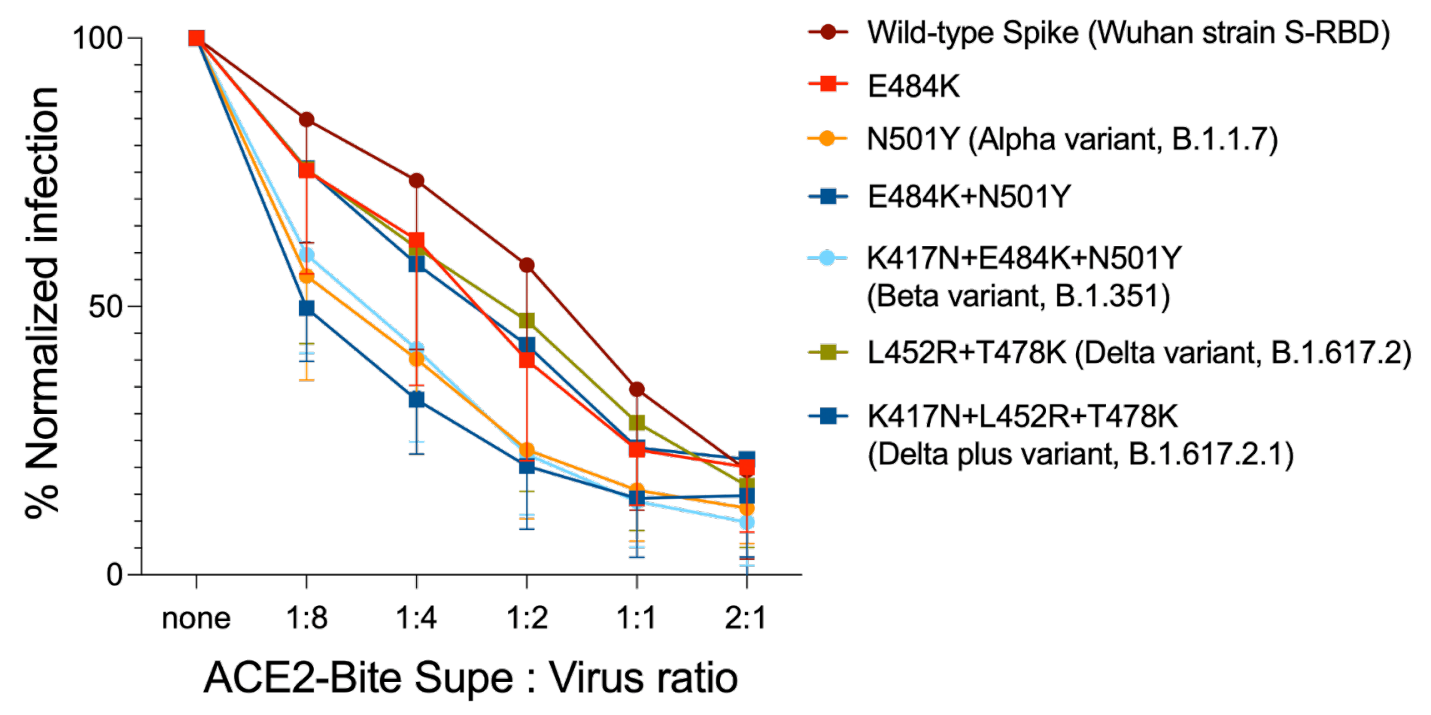


Supplementary Figure 1
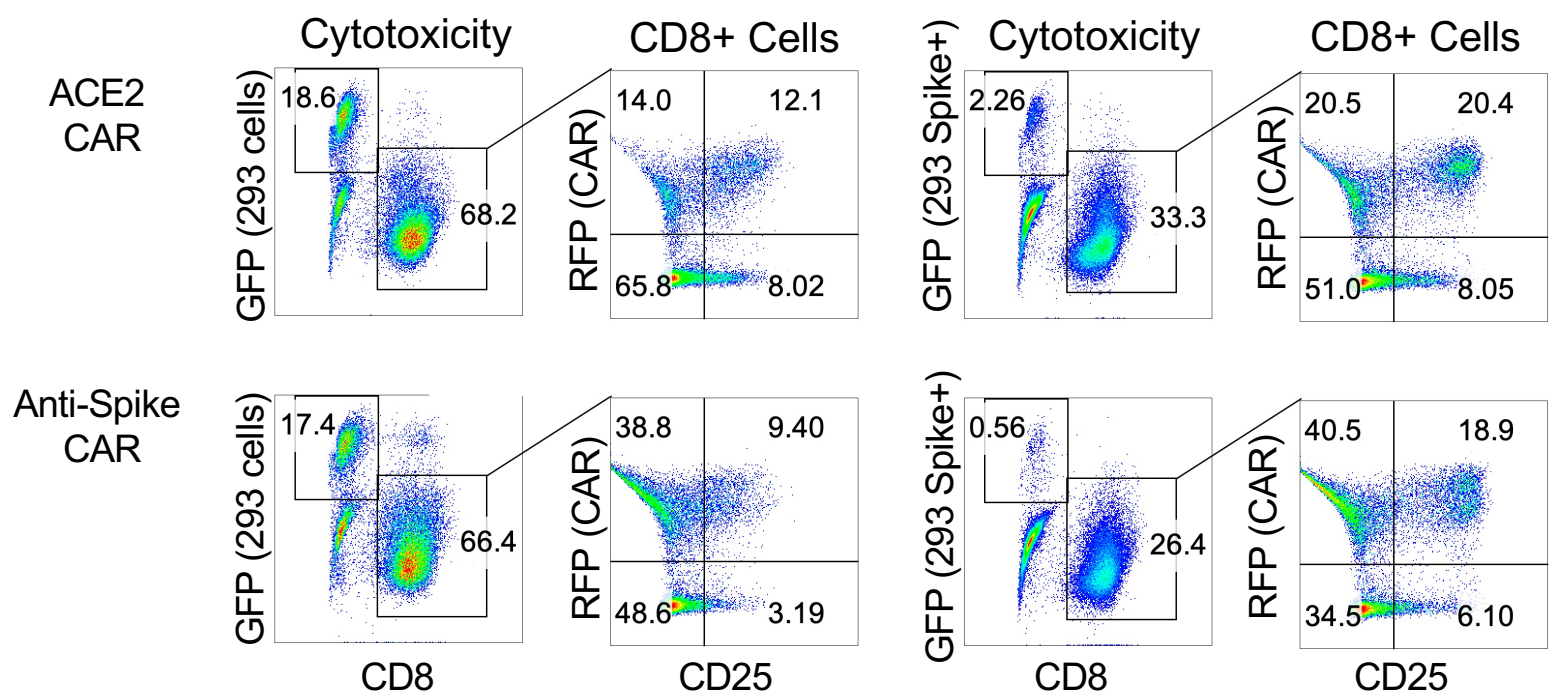
Supplementary Figure 2

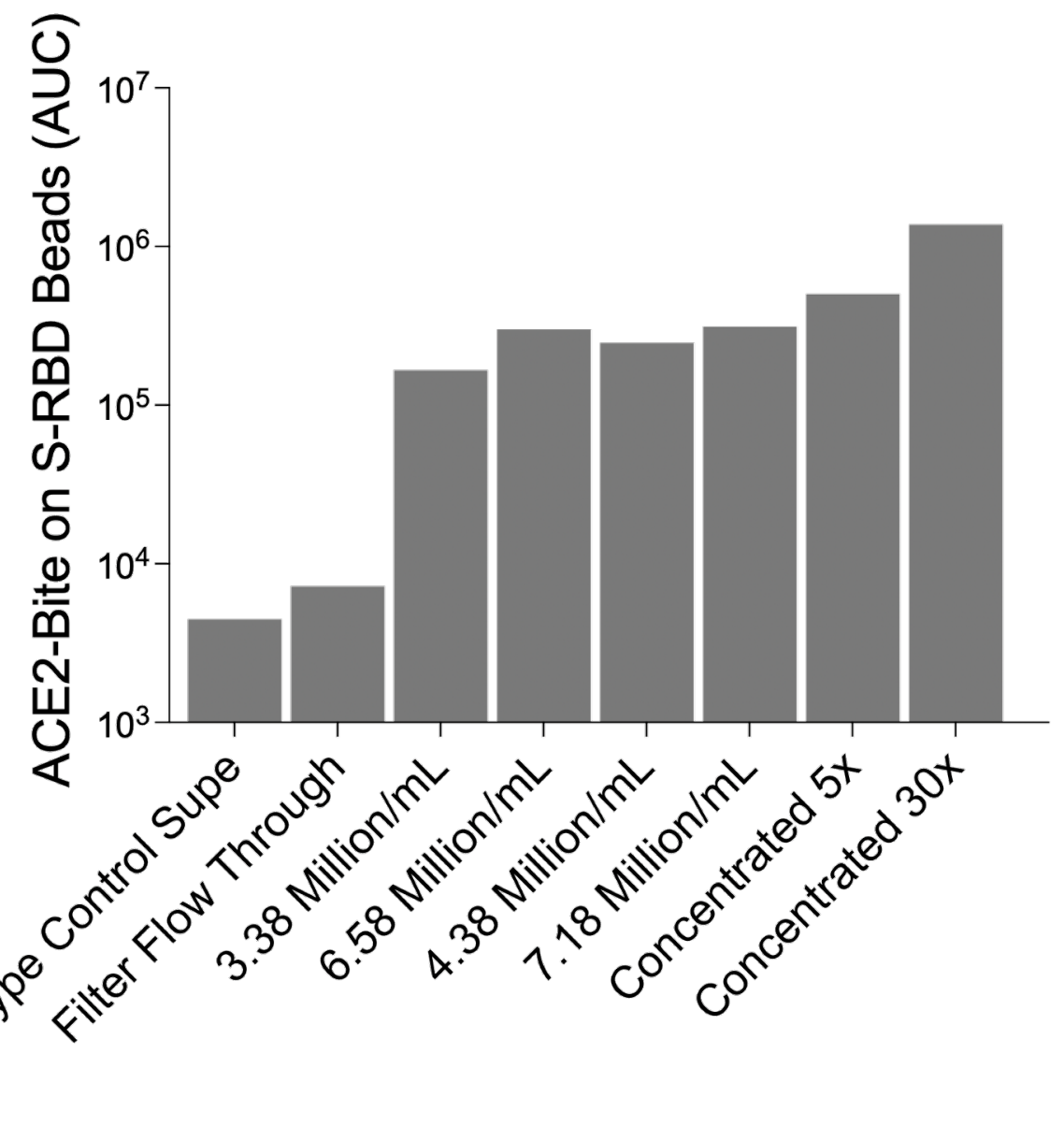

